\title{
A Two-Patch Predator-Prey Metapopulation Model
}

\author{
G. Quaglia, E. Re, M. Rinaldi and E. Venturino* \\ Dipartimento di Matematica "Giuseppe Peano", Università di Torino, \\ via Carlo Alberto 10, 10123 Torino, Italy. \\ Received 16 May 2012; Accepted (in revised version) 28 July 2012
}

Available online 23 August 2012

\begin{abstract}
A minimal model for predator-prey interaction in a composite environment is presented and analysed. We first consider free migrations between two patches for both interacting populations, and then the particular cases where only one-directional migration is allowed and where only one of the two populations can migrate. Our findings indicate that in all cases the ecosystem can never disappear entirely, under the model assumptions. The predator-free equilibrium and the coexistence of all populations are found to be the only feasible stable equilibria. When there are only one-directional migrations, the abandoned patch cannot be repopulated. Other equilibria then arise, with only prey in the second patch, coexistence in the second patch, or prey in both patches but predators only in the second one. For the case of sedentary prey, with predator migration, the prey cannot thrive alone in either of the two environments. However, predators can survive in a prey-free patch due to their ability to migrate into the other patch, provided prey is present there. If only the prey can migrate, the predators may be eliminated from one patch or from both. In the first case, the patch where there are no predators acts as a refuge for the survival of the prey.
\end{abstract}

AMS subject classifications: 92D25, 92D40

Key words: Complex ecosystems, fragmented habitats, migrations, population models, predatorprey, equilibria, stability.

\section{Introduction}

Habitat fragmentation is one of the major sources of biodiversity loss, which frequently occurs in nature due to natural causes or human activities [23]. A population originally thriving in an undisturbed environment may become partitioned into two or more patches (subpopulations) after a catastrophic event, and may continue to thrive independently in the particular patches where the living conditions are favourable. However, due to the reduced size or lesser resources of their smaller new environments they may then be extremely sensitive to adverse conditions, so that habitat fragmentation can ultimately lead

${ }^{*}$ Corresponding author. Email address: ezio.venturino@unito.it (E. Venturino) 
to species extinction. Metapopulation theory is an instrument devised to understand the associated dynamical processes [22]. One of its major achievements has been to demonstrate that in some circumstances a population can persist globally while the local populations become extinct $[5,9,11,22,24]$. Data collection, especially to assess migration rates, is not an activity generally undertaken by field scientists due to its intrinsic difficulty $[5,9]$ so the role of models is especially important [13]. Metapopulation models distinguish patches and paths by selecting the most favourable populated habitats as patches, with the remaining less populated ground in between regarded as inter-patch migration routes [10].

Successful examples where metapopulation dynamics has been applied are models for the mountain or bighorn sheep (Ovis canadensis) and the spotted owl (Strix occidentalis) [10]. The bighorn sheep Ovis canadensis has several predators - including the wolf (Canis lupus), coyote (Canis latrans), bear (Ursus), Canada lynx (Lynx canadensis), mountain lion (Puma concolor) and golden eagle (Aquila chrysaetos) [6]. Among the main predators of the spotted owl are the great horned owl Bubo virginianus - but at the same time Strix occidentalis hunts mainly small rodents, so can be viewed as an intermediate trophic level in a food web. Another example where the metapopulation approach to habitat fragmentation may be useful is the predator-prey system of the red fox Vulpes vulpes (L.) and rabbit Oryctolagus cuniculus (L.). Lepidoptera also play an important role in the development of Metapopulation theory. In Finland Melitaea cinxia has been studied using the concept of an incidence function [12], and also other species [16, 17]. Lepidoptera predators are mainly birds, bats, parasitoids, small mammals, reptiles and insects such as ants and dragonflies [2,3]. Mainly larvae are hunted; this occurs especially for some particular species such as Parus caerulens, P. major [4]. Genetic analysis tools have been used to prove that coral reef species may experience population reductions or extinctions at the local level [1]. These contribute to enhance overall meta-population genetic differentiations. A fairly recent review of modelling work on the consequences of habitat loss and fragmentation on interacting populations provides a set of testable hypotheses for experimentalists [18].

From the mathematical modelling viewpoint, nonlinear migrations depending on population growth rates that may lead to sustained population oscillations have been investigated [8]. Modifying the migration rates using concepts such as predator pursuit and prey evasion leads to reduced spatial synchrony and thus improved metapopulation persistence [14]. Explicit space dependence also leads to pattern formations in the metapopulation context [19]. A discrete version of metapopulation models, including a space description, is available [21]. An analytical formula to calculate the average lifetime of species living in fragmented habitats is provided in Ref. [7], which accounts for networks that are dynamically changing due to the destruction of patches and their reestablishment elsewhere in the landscape.

A recent investigation by one of the present authors concentrated on an extension of these metapopulation models to ecoepidemiology [15], when a disease is superimposed on a demographic system of interacting populations [20]. Inter alia, it was found that persistent oscillations in one patch can either carry through to a second patch or be dampened in the more composite environment, and in some cases the coexistence of all the populations 


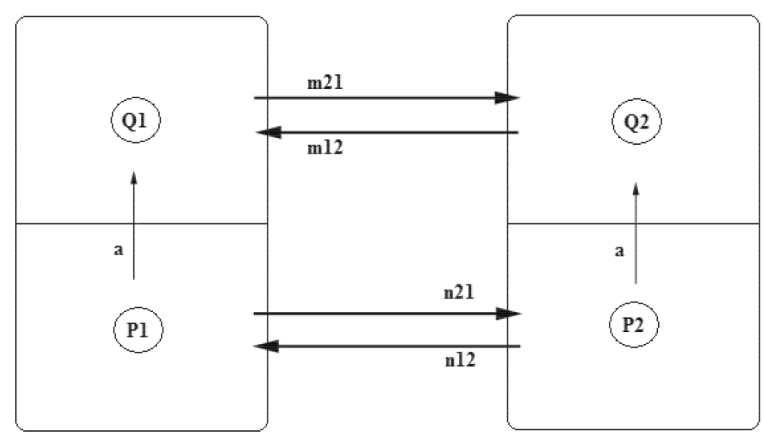

Figure 1: Illustration of the complete system (2.1).

in the metaecoepidemic model may be prevented. Further, the prey refuge at times may act as a reservoir that keeps the disease endemic, a negative consequence for the whole ecosystem.

In this article, we study a minimal model of demographic interaction type - viz. two populations, a prey and a predator, moving across two environmental patches. Several cases are envisioned. In the most general case, migrations are allowed back and forth for both populations. Specific disturbances are then allowed, where migrations can occur in one direction, or where only one of the populations migrates. In the next Section, the general model is presented and analysed. In Section 3, migrations in only one direction are allowed. Section 4 discusses the case where the prey cannot move from their patch, so that only predators migrate. In Section 5 we discuss the opposite case, where predators are confined to their environment but the prey can move back and forth between the patches. The concluding Section 6 discusses and compares the results.

\section{The Basic Model}

Let the prey population living in patch $i$ be denoted by $Q_{i}$ and the predator population living there by $P_{i}$, respectively. As illustrated in Fig. 1, it is assumed that each prey subpopulation thrives with the same reproduction rate but under different carrying capacities in each patch, and both subpopulations are subject to hunting by the predators. This is modelled via a simple mass action term. The predators do not have other food sources, so they are bound to starve if the prey is absent. This is expressed by a mortality rate. Unrestricted migrations to and from each patch are allowed for both the prey and the predators. Thus the resulting model equations are

$$
\begin{aligned}
& \dot{Q_{1}}=r Q_{1}\left(1-\frac{Q_{1}}{k_{1}}\right)-a Q_{1} P_{1}+m_{12} Q_{2}-m_{21} Q_{1}, \\
& \dot{Q_{2}}=r Q_{2}\left(1-\frac{Q_{2}}{k_{2}}\right)-a Q_{2} P_{2}-m_{12} Q_{2}+m_{21} Q_{1}, \\
& \dot{P_{1}}=-\mu_{1} P_{1}+a e Q_{1} P_{1}+n_{12} P_{2}-n_{21} P_{1}, \\
& \dot{P_{2}}=-\mu_{2} P_{2}+a e Q_{2} P_{2}-n_{12} P_{2}+n_{21} P_{1} .
\end{aligned}
$$


All of the parameters involved are assumed to be nonnegative and are as follows: $r$ denotes the prey reproduction rate, $k_{1}$ and $k_{2}$ the prey carrying capacities of patch 1 and 2 respectively, $a$ the predator hunting rate, $m_{i j}$ the migration rates of prey from patch $j$ into patch $i, \mu_{1}$ and $\mu_{2}$ the predator mortality rates in patch 1 and 2 respectively, $e$ the conversion factor of prey into new predators, and $n_{i j}$ are the predator migration rates from patch $j$ into patch $i$.

\subsection{Equilibria and Stability}

The system of equations (2.1) admits only three equilibria - viz. the origin $E^{(0)}=$ $(0,0,0,0)$, a predator-free equilibrium $E^{(1)}=\left(Q_{1}^{(1)}, Q_{2}^{(1)}, 0,0\right)$, and a coexistence equilib$\operatorname{rium} E^{(2)}=\left(Q_{1}^{(2)}, Q_{2}^{(2)}, P_{1}^{(2)}, P_{2}^{(2)}\right)$.

The predator-free equilibrium $E^{(1)}$ is determined as the intersection of two conic sections. More specifically, we need to solve the following system for the two parabolae:

$$
\Pi_{1}: Q_{2}=\frac{Q_{1}}{m_{12}}\left[m_{21}-r\left(1-\frac{Q_{1}}{k_{1}}\right)\right], \quad \Pi_{2}: Q_{1}=\frac{Q_{2}}{m_{21}}\left[m_{12}-r\left(1-\frac{Q_{2}}{k_{2}}\right)\right] .
$$

These parabolae both have roots at the origin and also at the two points $\left(\left(r-m_{21}\right) k_{1} r^{-1}, 0\right)$ and $\left(0,\left(r-m_{12}\right) k_{2} r^{-1}\right)$, respectively. Apart from at the origin, they intersect in at least one other point. There are four possible cases, depending on the signs of the roots. Thus $\Pi_{1}$ and $\Pi_{2}$ always intersect in the first quadrant when at least one of them is positive, and the only doubtful case is when both roots are negative. In that case, an intersection in the first quadrant exists if the slope at the origin of $\Pi_{2}$ is larger than that of $\Pi_{1}-$ i.e. $\Pi_{2}^{\prime}(0)>\Pi_{1}^{\prime}(0)$, which amounts to requiring that $m_{21}+m_{12}>r$ is trivially satisfied, since the negativity of the roots implies $m_{21}>r$ as well as $m_{12}>r$. Consequently, the predator-free equilibrium $E^{(1)}$ always exists and is unconditionally feasible.

For the coexistence equilibrium $E^{(2)}$, we find

$$
\begin{aligned}
Q_{1}^{(2)}= & \frac{\sigma-a e Q_{2}^{(2)}\left(\mu_{1}+n_{21}\right)}{a e\left(\mu_{2}+n_{12}-a e Q_{2}^{(2)}\right)}, \\
P_{1}^{(2)}= & \frac{1}{a n_{21}}\left(\mu_{2}+n_{12}-a e Q_{2}^{(2)}\right)\left(r-\frac{r}{k_{2}} Q_{2}^{(2)}-m_{12}\right) \\
& +\frac{m_{21}\left[\sigma-a e Q_{2}^{(2)}\left(\mu_{1}+n_{21}\right)\right]}{a^{3} e^{2} n_{21} Q_{2}^{(2)}}, \\
P_{2}^{(2)}= & \frac{1}{a}\left(r-\frac{r}{k_{2}} Q_{2}^{(2)}-m_{12}\right)+\frac{m_{21}\left[\sigma-a e Q_{2}^{(2)}\left(\mu_{1}+n_{21}\right)\right]}{a^{3} e^{2} Q_{2}^{(2)}\left(\mu_{2}+n_{12}-a e Q_{2}^{(2)}\right)} .
\end{aligned}
$$


Here $\sigma=\mu_{1} \mu_{2}+\mu_{1} n_{12}+\mu_{2} n_{21}$ and $Q_{2}^{(2)}$ satisfies the equation of fifth degree

$$
\begin{aligned}
& \operatorname{raek}_{1} n_{21} \Phi \Omega^{2} Q_{2}-r n_{21} \Phi^{2} Q_{2}+a^{2} e^{2} k_{1} m_{12} n_{21} \Omega^{2} Q_{2}^{2}-a^{2} e^{2} k_{1} m_{21} n_{21} \Phi \Omega Q_{2} \\
& -\operatorname{aek}_{1} \Phi \Omega^{2} Q_{2}\left(r-\frac{r}{k_{2}} Q_{2}-m_{12}\right)+k_{1} m_{21} \Phi^{2} \Omega=0
\end{aligned}
$$

with $\Phi\left(Q_{2}\right)=\sigma-a e Q_{2}\left(\mu_{1}+n_{21}\right)$ and $\Omega\left(Q_{2}\right)=\mu_{2}+n_{12}-a e Q_{2}$, from which we require a nonnegative root. It turns out that Eq. (2.3) can be rewritten as

$$
\sum_{k=0}^{5} A_{k} Q_{2}^{k}=0, \quad A_{0}=k_{1} m_{21} \sigma^{2}\left(\mu_{2}+n_{12}\right), \quad A_{5}=-a^{4} e^{4} k_{1} \frac{r}{k_{2}}\left(\mu_{1}+n_{21}\right),
$$

so we may conclude that there is at least one positive root - i.e. at least one feasible equilibrium must exist. (We do not investigate uniqueness.) Further feasibility conditions in this case come from Eq. (2.2), which can be rewritten more compactly as

$$
P_{2}^{(2)}=\frac{1}{a}\left[r-\frac{r}{k_{2}} Q_{2}^{(2)}-m_{12}+\frac{m_{21}}{a e Q_{2}^{(2)}} Q_{1}^{(2)}\right], \quad P_{1}^{(2)}=\frac{1}{n_{21}}\left[\mu_{2}+n_{12}-a e Q_{2}^{(2)}\right] P_{2}^{(2)}
$$

where we require

$$
\mu_{2}+n_{12} \geq a e Q_{2}^{(2)}
$$

so the other non-negativity conditions for $Q_{1}^{(2)}$ and $P_{2}^{(2)}$ follow - viz.

$$
\sigma \geq a e Q_{2}^{(2)}\left(\mu_{1}+n_{21}\right), \quad r+\frac{m_{21}}{a e Q_{2}^{(2)}} Q_{1}^{(2)} \geq \frac{r}{k_{2}} Q_{1}^{(2)}+m_{12} .
$$

The stability hinges on the Jacobian of Eq. (2.1) - i.e.

$$
J=\left(\begin{array}{cccc}
J_{11} & m_{12} & -a Q_{1} & 0 \\
m_{21} & J_{22} & 0 & -a Q_{2} \\
a e P_{1} & 0 & J_{33} & n_{12} \\
0 & a e P_{2} & n_{21} & J_{44}
\end{array}\right),
$$

where

$$
\begin{array}{ll}
J_{11}=r\left(1-2 \frac{Q_{1}}{k_{1}}\right)-a P_{1}-m_{21}, & J_{22}=r\left(1-2 \frac{Q_{2}}{k_{2}}\right)-a P_{2}-m_{12}, \\
J_{33}=-\mu_{1}+a e Q_{1}-n_{21}, & J_{44}=-\mu_{2}+a e Q_{2}-n_{12} .
\end{array}
$$

At the origin $E^{(0)}$ the characteristic equation factors into the product of two quadratic equations, yielding two pairs of eigenvalues. The first pair is

$$
\begin{aligned}
& \lambda_{1}=\frac{1}{2}\left(-\mu_{1}-\mu_{2}-n_{12}-n_{21}-\sqrt{\Delta_{1}}\right)<0, \\
& \lambda_{2}=\frac{1}{2}\left(-\mu_{1}-\mu_{2}-n_{12}-n_{21}+\sqrt{\Delta_{1}}\right)
\end{aligned}
$$


with $\Delta_{1}=\left(J_{22}^{(0)}+J_{44}^{(0)}\right)^{2}-4 J_{22}^{(0)} J_{44}^{(0)}+4 n_{12} n_{21}$ and $J_{11}^{(0)}=r-m_{21}, J_{22}^{(0)}=-\mu_{1}-n_{21}, J_{33}^{(0)}=r-m_{12}$, $J_{44}^{(0)}=-\mu_{2}-n_{12}$. We also find that $\operatorname{Re}\left(\lambda_{2}\right)<0$ for $\Delta_{1}<0$, for $\Delta_{1}=0$ the result $\lambda_{2}<0$ is trivial, and for $\Delta_{1}>0$ the negative eigenvalue $\lambda_{2}<0$ becomes $\mu_{1} \mu_{2}+\mu_{1} n_{12}+\mu_{2} n_{21}>0$ that holds unconditionally. For the second pair we have

$$
\begin{aligned}
& \lambda_{3}=\frac{1}{2}\left(2 r-m_{12}-m_{21}-\sqrt{\Delta_{2}}\right), \\
& \lambda_{4}=\frac{1}{2}\left(2 r-m_{12}-m_{21}+\sqrt{\Delta_{2}}\right)
\end{aligned}
$$

with $\Delta_{2}=\left(J_{11}^{(0)}+J_{33}^{(0)}\right)^{2}-4 J_{11}^{(0)} J_{33}^{(0)}+4 m_{12} m_{21}=\left(m_{12}+m_{21}\right)^{2}$ such that $\lambda_{3}=r>0$ and $\lambda_{4}=r-m_{12}-m_{21}$, thereby showing the unconditional instability of the origin.

At the predator-free equilibrium $E^{(1)}$, the characteristic equation factors into the product of two quadratic equations. The first is

$$
\lambda^{2}-\left(J_{11}^{(1)}+J_{33}^{(1)}\right) \lambda+J_{11}^{(1)} J_{33}^{(1)}-m_{12} m_{21}=0,
$$

when the Routh-Hurtwitz conditions

$$
J_{11}^{(1)}+J_{33}^{(1)}<0 \text { and }-m_{12} m_{21}+J_{11}^{(1)} J_{33}^{(1)}>0
$$

lead to the first set of stability conditions

$$
\begin{aligned}
& 2 r<2 r\left(\frac{Q_{1}^{(1)}}{k_{1}}+\frac{Q_{2}^{(1)}}{k_{2}}\right)+m_{12}+m_{21}, \\
& r-m_{12}-m_{21}+4 \frac{Q_{1}^{(1)} Q_{2}^{(1)}}{k_{1} k_{2}}>2 \frac{Q_{1}^{(1)}}{k_{1}}\left(r-m_{12}\right)+2 \frac{Q_{2}^{(1)}}{k_{2}}\left(r-m_{21}\right) .
\end{aligned}
$$

The second quadratic is

$$
\lambda^{2}-\left(J_{22}^{(1)}+J_{44}^{(1)}\right) \lambda+J_{22}^{(1)} J_{44}^{(1)}-n_{12} n_{21}=0,
$$

when the Routh-Hurwitz conditions yield

$$
\begin{aligned}
& Q_{1}^{(1)}+Q_{2}^{(1)}<\frac{\mu_{1}+\mu_{2}+n_{12}+n_{21}}{a e}, \\
& \sigma+a^{2} e^{2} Q_{1}^{(1)} Q_{2}^{(1)}>a e\left[Q_{1}^{(1)}\left(\mu_{2}+n_{12}\right)+Q_{2}^{(1)}\left(\mu_{1}+n_{21}\right)\right] .
\end{aligned}
$$

At the coexistence equilibrium $E^{(2)}$, the characteristic equation $\sum_{i=0}^{4} \lambda^{i} a_{i}=0$ has rather complicated coefficients - viz.

$$
\begin{aligned}
a_{3}= & -J_{11}^{(2)}-J_{22}^{(2)}-J_{33}^{(2)}-J_{44}^{(2)}, \\
a_{2}= & J_{11}^{(2)} J_{22}^{(2)}+J_{11}^{(2)} J_{33}^{(2)}+J_{11}^{(2)} J_{44}^{(2)}+J_{22}^{(2)} J_{33}^{(2)}+J_{22}^{(2)} J_{44}^{(2)}+J_{33}^{(2)} J_{44}^{(2)}+a^{2} e\left(Q_{1}^{(2)} P_{1}^{(2)}+Q_{2}^{(2)} P_{2}^{(2)}\right) \\
& -n_{12} n_{21}-m_{12} m_{21} \\
a_{1}= & -J_{11}^{(2)} J_{22}^{(2)} J_{33}^{(2)}-J_{11}^{(2)} J_{22}^{(2)} J_{44}^{(2)}-J_{11}^{(2)} J_{33}^{(2)} J_{44}^{(2)}-J_{22}^{(2)} J_{33}^{(2)} J_{44}^{(2)}-a^{2} e\left[Q_{2}^{(2)} P_{2}^{(2)}\left(J_{11}^{(2)}+J_{22}^{(2)}\right)\right. \\
& \left.+Q_{1}^{(2)} P_{1}^{(2)}\left(J_{33}^{(2)}+J_{44}^{(2)}\right)\right]+n_{12} n_{21}\left(J_{11}^{(2)}+J_{33}^{(2)}\right)+m_{12} m_{21}\left(J_{22}^{(2)}+J_{44}^{(2)}\right) \\
a_{0}= & J_{11}^{(2)} J_{22}^{(2)} J_{33}^{(2)} J_{44}^{(2)}+a^{2} e\left(m_{21} n_{12} Q_{1}^{(2)} P_{2}^{(2)}+m_{12} n_{21} Q_{2}^{(2)} P_{1}^{(2)}+a^{2} e Q_{1}^{(2)} Q_{2}^{(2)} P_{1}^{(2)} P_{2}^{(2)}\right. \\
& \left.+Q_{1}^{(2)} P_{1}^{(2)} J_{33}^{(2)} J_{44}^{(2)}+Q_{2}^{(2)} P_{2}^{(2)} J_{11}^{(2)} J_{22}^{(2)}\right)-m_{12} m_{21} J_{22}^{(2)} J_{44}^{(2)}-n_{12} n_{21} J_{11}^{(2)} J_{33}^{(2)} .
\end{aligned}
$$


The four stability conditions can still be written down explicitly but are quite involved:

$$
\begin{aligned}
& J_{11}^{(2)}+J_{22}^{(2)}+J_{33}^{(2)}+J_{44}^{(2)}<0, \\
& J_{11}^{(2)} J_{22}^{(2)} J_{33}^{(2)} J_{44}^{(2)}+a^{2} e\left(m_{21} n_{12} Q_{1}^{(2)} P_{2}^{(2)}+m_{12} n_{21} Q_{2}^{(2)} P_{1}^{(2)}+a^{2} e Q_{1}^{(2)} Q_{2}^{(2)} P_{1}^{(2)} P_{2}^{(2)}+Q_{1}^{(2)} P_{1}^{(2)} J_{33}^{(2)} J_{44}^{(2)}\right. \\
& \left.+Q_{2}^{(2)} P_{2}^{(2)} J_{11}^{(2)} J_{22}^{(2)}\right)>m_{12} m_{21} J_{22}^{(2)} J_{44}^{(2)}+n_{12} n_{21} J_{11}^{(2)} J_{33}^{(2)} \text {, } \\
& {J_{11}^{(2)}}^{2}\left(J_{22}^{(2)}+J_{33}^{(2)}+J_{44}^{(2)}\right)+J_{22}^{(2)}\left(J_{11}^{(2)}+J_{33}^{(2)}+J_{44}^{(2)}\right)+J_{33}^{(2) 2}\left(J_{11}^{(2)}+J_{22}^{(2)}+J_{44}^{(2)}\right) \\
& +J_{44}^{(2)}\left(J_{11}^{(2)}+J_{22}^{(2)}+J_{33}^{(2)}\right)+2 J_{11}^{(2)} J_{22}^{(2)} J_{33}^{(2)}+2 J_{11}^{(2)} J_{22}^{(2)} J_{44}^{(2)}+2 J_{11}^{(2)} J_{33}^{(2)} J_{44}^{(2)}+2 J_{22}^{(2)} J_{33}^{(2)} J_{44}^{(2)} \\
& +a^{2} e Q_{1}^{(2)} P_{1}^{(2)}\left(J_{11}^{(2)}+J_{22}^{(2)}\right)+a^{2} e Q_{2}^{(2)} P_{2}^{(2)}\left(J_{33}^{(2)}+J_{44}^{(2)}\right)-m_{12} m_{21}\left(J_{11}^{(2)}+J_{33}^{(2)}\right) \\
& -n_{12} n_{21}\left(J_{22}^{(2)}+J_{44}^{(2)}\right)<0, \\
& \left\{J_{11}^{(2)} J_{22}^{(2)} J_{33}^{(2)}+J_{11}^{(2)} J_{22}^{(2)} J_{44}^{(2)}+J_{11}^{(2)} J_{33}^{(2)} J_{44}^{(2)}+J_{22}^{(2)} J_{33}^{(2)} J_{44}^{(2)}+a^{2} e\left[Q_{1}^{(2)} P_{1}^{(2)}\left(J_{33}^{(2)}+J_{44}^{(2)}\right)\right.\right. \\
& \left.\left.+Q_{2}^{(2)} P_{2}^{(2)}\left(J_{11}^{(2)}+J_{22}^{(2)}\right)\right]-m_{12} m_{21}\left(J_{22}^{(2)}+J_{44}^{(2)}\right)-n_{12} n_{21}\left(J_{11}^{(2)}+J_{33}^{(2)}\right)\right\} \\
& \times\left[J_{11}^{(2)}{ }^{2}\left(J_{22}^{(2)}+J_{33}^{(2)}+J_{44}^{(2)}\right)+J_{22}^{(2)}{ }^{2}\left(J_{11}^{(2)}+J_{33}^{(2)}+J_{44}^{(2)}\right)+J_{33}^{(2)} 2\left(J_{11}^{(2)}+J_{22}^{(2)}+J_{44}^{(2)}\right)\right. \\
& +J_{44}^{(2)}\left(J_{11}^{(2)}+J_{22}^{(2)}+J_{33}^{(2)}\right)+2 J_{11}^{(2)} J_{22}^{(2)} J_{33}^{(2)}+2 J_{11}^{(2)} J_{22}^{(2)} J_{44}^{(2)}+2 J_{11}^{(2)} J_{33}^{(2)} J_{44}^{(2)}+2 J_{22}^{(2)} J_{33}^{(2)} J_{44}^{(2)} \\
& +J_{11}^{(2)}\left(a^{2} e Q_{1}^{(2)} P_{1}^{(2)}-m_{12} m_{21}\right)+J_{22}^{(2)}\left(a^{2} e Q_{1}^{(2)} P_{1}^{(2)}-n_{12} n_{21}\right)+J_{33}^{(2)}\left(a^{2} e Q_{2}^{(2)} P_{2}^{(2)}-m_{12} m_{21}\right) \\
& \left.+J_{44}^{(2)}\left(a^{2} e Q_{2}^{(2)} P_{2}^{(2)}-n_{12} n_{21}\right)\right]>\left[J_{11}^{(2)} J_{22}^{(2)} J_{33}^{(2)} J_{44}^{(2)}+a^{2} e\left(m_{21} n_{12} Q_{1}^{(2)} P_{2}^{(2)}+m_{12} n_{21} Q_{2}^{(2)} P_{1}^{(2)}\right.\right. \\
& \left.\left.+a^{2} e Q_{1}^{(2)} Q_{2}^{(2)} P_{1}^{(2)} P_{2}^{(2)}+Q_{1}^{(2)} P_{1}^{(2)} J_{33}^{(2)} J_{44}^{(2)}+Q_{2}^{(2)} P_{2}^{(2)} J_{11}^{(2)} J_{22}^{(2)}\right)-m_{12} m_{21} J_{22}^{(2)} J_{44}^{(2)}-n_{12} n_{21} J_{11}^{(2)} J_{33}^{(2)}\right] \\
& \times\left(J_{11}^{(2)}+J_{22}^{(2)}+J_{33}^{(2)}+J_{44}^{(2)}\right)^{2} \text {. }
\end{aligned}
$$

A simulation for the parameter values $r=8, k_{1}=100, k_{2}=150, a=0.5, m_{12}=2$, $m_{21}=3, \mu_{1}=3, \mu_{2}=4, e=0.3, n_{12}=2.5, n_{21}=2.8$ shows that this equilibrium can be achieved - cf. Fig. 2 . We also illustrate another simulation leading to a prey-only equilibrium, obtained with the parameters $r=6, k_{1}=100, k_{2}=150, a=0.5, m_{12}=2$, $m_{21}=3, \mu_{1}=10, \mu_{2}=14, e=0.2, n_{12}=2.5, n_{21}=2.8-$ cf. Fig. 3 .

\section{Migrations in One Direction Only}

We now set $m_{12}=n_{12}=0$ in Eq. (2.1), to consider the system describing migrations in only one direction:

$$
\begin{aligned}
& \dot{Q_{1}}=r Q_{1}\left(1-\frac{Q_{1}}{k_{1}}\right)-a Q_{1} P_{1}-m_{21} Q_{1}, \\
& \dot{Q_{2}}=r Q_{2}\left(1-\frac{Q_{2}}{k_{2}}\right)-a Q_{2} P_{2}+m_{21} Q_{1},
\end{aligned}
$$



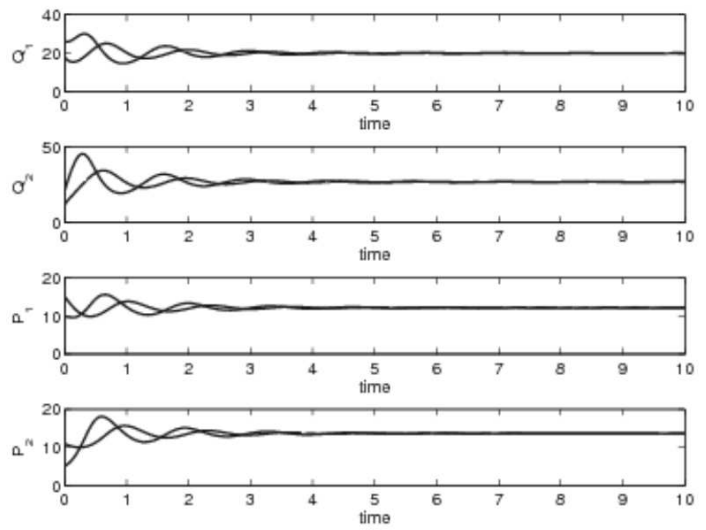

Figure 2: Coexistece equilibrium $E^{(2)}$, obtained for the parameter values $r=8, k_{1}=100, k_{2}=150$, $a=0.5, m_{12}=2, m_{21}=3, \mu_{1}=3, \mu_{2}=4, e=0.3, n_{12}=2.5, n_{21}=2.8$.
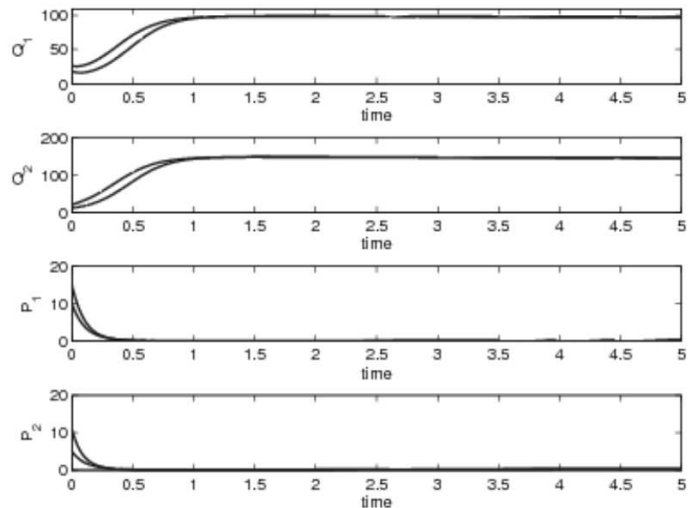

Figure 3: Prey-only equilibrium $E^{(1)}$, obtained for the parameter values $r=6, k_{1}=100, k_{2}=150$, $a=0.5, m_{12}=2, m_{21}=3, \mu_{1}=10, \mu_{2}=14, e=0.2, n_{12}=2.5, n_{21}=2.8$.

$$
\begin{aligned}
& \dot{P_{1}}=P_{1}\left(-\mu_{1}+a e Q_{1}-n_{21}\right), \\
& \dot{P_{2}}=-\mu_{2} P_{2}+a e Q_{2} P_{2}+n_{21} P_{1} .
\end{aligned}
$$

The scheme for this model is illustrated in Fig. 4.

\subsection{Equilibria}

The equilibria are now the origin $X^{(0)}$, the boundary points $X^{(1)}=\left(0, k_{2}, 0,0\right)$ and $X^{(2)}=\left(0, Q_{2}^{(2)}, 0, P_{2}^{(2)}\right)$ with

$$
Q_{2}^{(2)}=\frac{\mu_{2}}{a e}, \quad P_{2}^{(2)}=\frac{r}{a}\left(1-\frac{\mu_{2}}{k_{2} a e}\right),
$$




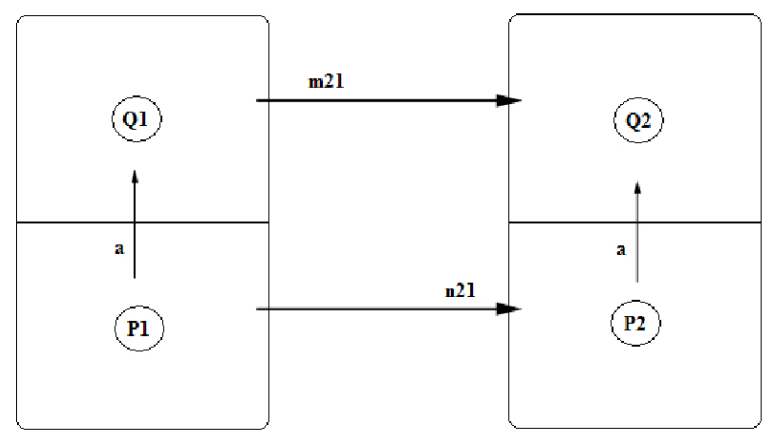

Figure 4: Graphical illustration of the system (3.1).

also $X^{(3)}=\left(Q_{1}^{(3)}, Q_{2}^{(3)}, 0,0\right)$ with

$$
Q_{1}^{(3)}=k_{1}-\frac{m_{21}}{r} k_{1}, \quad Q_{2}^{(3)}=\frac{k_{2}}{2}\left[1+\sqrt{1+4 m_{21} \frac{k_{1}}{r k_{2}}\left(1-\frac{m_{21}}{r}\right)}\right],
$$

and finally $X^{(4)}=\left(Q_{1}^{(4)}, Q_{2}^{(4)}, 0, P_{2}^{(4)}\right)$ with population values

$$
\begin{aligned}
& Q_{1}^{(4)}=k_{1}-\frac{m_{21}}{r} k_{1}, \quad Q_{2}^{(4)}=\frac{\mu_{2}}{a e}, \\
& P_{2}^{(4)}=\frac{r}{a}\left(1-\frac{\mu_{2}}{a e k_{2}}\right)-\frac{e}{\mu_{2}}\left(m_{21} k_{1}-\frac{m_{21}^{2} k_{1}}{r}\right) .
\end{aligned}
$$

Obviously, $X^{(2)}$ is feasible if

$$
a e k_{2} \geq \mu_{2}
$$

and $X^{(3)}$ is feasible if

$$
r \geq m_{21}
$$

The feasibility conditions for $X^{(4)}$ are

$$
r \geq m_{21}, \quad r^{2} \mu_{2}\left(a e k_{2}-\mu_{2}\right)+a^{2} e^{2} k_{1} k_{2} m_{21}\left(r-m_{21}\right) \geq 0 .
$$

After solving for the two population values $P_{1}^{(5)}$ and $Q_{1}^{(5)}$ in the steady state system, the coexistence equilibrium $X^{(5)}=\left(Q_{1}^{(5)}, Q_{2}^{(5)}, P_{1}^{(5)}, P_{2}^{(5)}\right)$ follows from determining the intersection of the following two hyperbolae in the first quadrant of the $Q_{2}-P_{2}$ phase plane:

$$
\varphi_{1}: D Q_{2}-D Q_{2}^{2}-a Q_{2} P_{2}+E=0 \quad \text { and } \quad \varphi_{2}: P_{2}=\frac{A}{B Q_{2}-C},
$$

where

$$
\begin{gathered}
A=n_{21}\left[m_{21}-r\left(1-\frac{\mu_{1}+n_{21}}{k_{1} a e}\right)\right], \quad B=a^{2} e, \\
C=a \mu_{2}, \quad D=\frac{r}{k_{2}}, \quad E=\frac{m_{21}}{a e}\left(\mu_{1}+n_{21}\right) .
\end{gathered}
$$


The remaining populations have the values

$$
P_{1}^{(5)}=\frac{r}{a}\left(1-\frac{\mu_{1}+n_{21}}{k_{1} a e}\right)-\frac{m_{21}}{a}, \quad Q_{1}^{(5)}=\frac{\mu_{1}+n_{21}}{a e},
$$

such that $X^{(5)}$ is feasible if

$$
k_{1} a e\left(r-m_{21}\right)-r\left(\mu_{1}+n_{21}\right)>0 .
$$

To study the hyperbolae (3.5), we observe that

$$
A=-\frac{n_{21}}{a e k_{1}}\left[a e k_{1}\left(r-m_{21}\right)-r\left(\mu_{1}+n_{21}\right)\right]<0
$$

if we invoke Eq. (3.6), and all the other coefficients are nonnegative. Now $\varphi_{1}$ has the vertical asymptote $Q_{2}=0$ and also the asymptote $P_{2}=D a^{-1}\left(1-Q_{2}\right)$, and $\varphi_{2}$ has the asymptotes $P_{2}=0$ and $Q_{2}=C B^{-1}=\mu_{2}(a e)^{-1}>0$. Thus there always exists an intersection in the first quadrant since $A<0$, and since

$$
\frac{C}{B} \rightarrow 0, \quad \frac{D}{a} \rightarrow \infty
$$

this intersection tends to $(0,+\infty)-$ cf. also Fig. 5 . Thus the coexistence equilibrium is feasible if the inequality (3.6) holds.

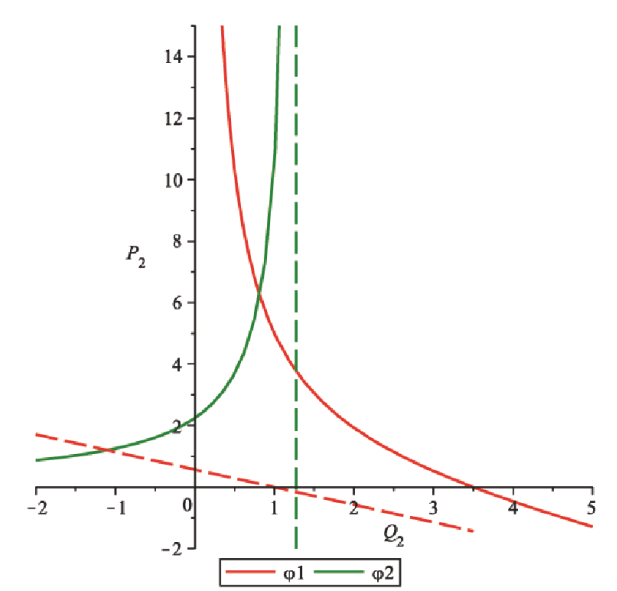

Figure 5: The intersection of the two hyperbolae, for the parameter values $a=0.7, A=-0.2, B=0.07$, $C=0.089, D=0.4, E=3.5$.

\subsection{Stability}

The Jacobian of Eq. (3.1) is

$$
J=\left(\begin{array}{cccc}
J_{11} & 0 & -a Q_{1} & 0 \\
m_{21} & r-2 \frac{r}{k_{2}} Q_{2}-a P_{2} & 0 & -a Q_{2} \\
a e P_{1} & 0 & -\mu_{1}+a e Q_{1}-n_{21} & 0 \\
0 & a e P_{2} & n_{21} & -\mu_{2}+a e Q_{2}
\end{array}\right),
$$


where

$$
J_{11}=r\left(1-\frac{Q_{1}}{k_{1}}\right)-a P_{1}-m_{21}-\frac{r}{k_{1}} Q_{1} .
$$

At the origin $X^{(0)}$, the eigenvalues are $\lambda_{1}=-\mu_{1}-n_{21}<0, \lambda_{2}=-\mu_{2}<0, \lambda_{3}=r-m_{21}$ and $\lambda_{4}=r>0$, implying unconditional instability. At $X^{(1)}$, we find $\lambda_{1}=r-m_{21}, \lambda_{2}=-r<0$, $\lambda_{3}=-\mu_{1}-n_{21}<0$ and $\lambda_{4}=-\mu_{2}+a e k_{2}$, so this equilibrium is stable if

$$
r<m_{21}, \quad a e k_{2}<\mu_{2} .
$$

At $X^{(2)}$, we have the two explicit eigenvalues $\lambda_{1}=r-m_{21}$ and $\lambda_{2}=-\mu_{1}-n_{21}<0$, and the others are roots of the quadratic

$$
\lambda^{2}+\frac{r \mu_{2}}{k_{2} a e} \lambda+\mu_{2} r\left(1-\frac{\mu_{2}}{k_{2} a e}\right)=0
$$

- i.e.

$$
\begin{aligned}
& \lambda_{3}=\frac{-r \mu_{2}-\sqrt{r^{2} \mu_{2}^{2}+4 k_{2} a e \mu_{2} r\left(-k_{2} a e+\mu_{2}\right)}}{2 k_{2} a e}<0, \\
& \lambda_{4}=\frac{-r \mu_{2}+\sqrt{r^{2} \mu_{2}^{2}+4 k_{2} a e \mu_{2} r\left(-k_{2} a e+\mu_{2}\right)}}{2 k_{2} a e} .
\end{aligned}
$$

Further, we have $\lambda_{4}<0$ from inequality (3.2), so there is stability provided

$$
r<m_{21} \text {. }
$$

At $X^{(3)}$, once again all the eigenvalues are immediate — viz.

$$
\begin{array}{ll}
\lambda_{1}=-r+m_{21}<0, & \lambda_{2}=-r \sqrt{\xi}<0, \\
\lambda_{3}=\operatorname{aek}_{1}\left(1-\frac{m_{21}}{r}\right)-\mu_{1}-n_{21}, & \lambda_{4}=\frac{a e}{2} k_{2} \sqrt{\xi}-\mu_{2},
\end{array}
$$

where

$$
\xi=1+4 \frac{m_{21} k_{1}}{r k_{2}}\left(1-\frac{m_{21}}{r}\right) .
$$

After some algebra, the last two eigenvalues yield the stability conditions

$$
\begin{aligned}
& r a e k_{1}<r\left(\mu_{1}+n_{21}\right)+a e k_{1} m_{21}, \\
& r a^{2} e^{2} k_{1} k_{2} m_{21}+r^{2} a e k_{2} \mu_{2}<\mu_{2}^{2} r^{2}+a^{2} e^{2} k_{1} k_{2} m_{21}^{2} .
\end{aligned}
$$

The Jacobian evaluated at the equilibrium $X^{(4)}$ again provides two explicit eigenvalues viz. $\lambda_{1}=-r+m_{21}$, which is negative in view of the feasibility condition (3.4), and

$$
\lambda_{2}=-\mu_{1}+a e k_{1}\left(1-\frac{m_{21}}{r}\right)-n_{21}=\frac{1}{r}\left(-r \mu_{1}-r n_{21}-a e k_{1} m_{21}+a e k_{1} r\right),
$$


which is negative if $a e k_{1}\left(r-m_{21}\right)<r\left(\mu_{1}+n_{21}\right)$. The others are roots of a quadratic for which the Routh-Hurwitz conditions are always satisfied. Indeed, $J_{22}^{(4)}<0$ reduces to $r^{2} \mu_{2}^{2}+a^{2} e^{2} k_{1} k_{2} m_{21}\left(r-m_{21}\right)>0$, which is always true from (3.4), and the other is simply $\mu_{2} a P_{2}^{(4)}>0$. Consequently, there is stability if

$$
\operatorname{aek}_{1}\left(r-m_{21}\right)<r\left(\mu_{1}+n_{21}\right) .
$$

For the coexistence equilibrium, the characteristic equation factors into the product of two quadratic equations

$$
a^{2} e P_{1}^{(5)} Q_{1}^{(5)}-\lambda\left(J_{11}^{(5)}-\lambda\right)=0, \quad\left(J_{22}^{(5)}-\lambda\right)\left(J_{44}^{(5)}-\lambda\right)+a^{2} e P_{2}^{(5)} Q_{2}^{(5)}=0,
$$

such that the Routh-Hurwitz conditions for the first yield the inequalities

$$
\frac{r}{k_{1} a e}\left(\mu_{1}+n_{21}\right)>0, \quad a^{2} e Q_{1}^{(5)} P_{1}^{(5)}>0
$$

that are always satisfied, and they reduce to $J_{22}^{(5)}+J_{44}^{(5)}<0$ and $J_{22}^{(5)} J_{44}^{(5)}+a^{2} e P_{2}^{(5)} Q_{2}^{(5)}>0$ for the second. Thus we obtain the stability conditions

$$
\begin{aligned}
& r-\frac{2 r}{k_{2}} Q_{2}^{(5)}-a P_{2}^{(5)}-\mu_{2}+a e Q_{2}^{(5)}<0, \\
& r\left(\mu_{1}+n_{21}\right)\left(\mu_{2}-a e Q_{2}^{(5)}\right)+a^{3} e^{2} k_{1} Q_{2}^{(5)} P_{2}^{(5)}>0 .
\end{aligned}
$$

In Fig. 6, we verify that the feasibility conditions (3.4) and the stability conditions (3.10) are not empty when $r=8, k_{1}=100, k_{2}=150, a=0.5, m_{12}=0, m_{21}=3, \mu_{1}=6$, $\mu_{2}=3, e=0.3, n_{12}=0, n_{21}=2.8$. In Fig. 7 , for the coexistence equilibrium we verify that the feasibility condition (3.6) and the conditions (3.11) and (3.12) for stability are indeed achievable, on choosing $r=8, k_{1}=100, k_{2}=150, a=0.5, m_{12}=0, m_{21}=3$, $\mu_{1}=3, \mu_{2}=4, e=0.3, n_{12}=0$, and $n_{21}=2.8$.

\section{Only Predators Migrate}

We now set $m_{12}=m_{21}=0$ to consider the case when there is only predator migration, as represented in Fig. 8. The system of equations then reduces to

$$
\begin{aligned}
& \dot{Q_{1}}=Q_{1}\left[r\left(1-\frac{Q_{1}}{k_{1}}\right)-a P_{1}\right], \\
& \dot{Q_{2}}=Q_{2}\left[r\left(1-\frac{Q_{2}}{k_{2}}\right)-a P_{2}\right], \\
& \dot{P_{1}}=-\mu_{1} P_{1}+a e Q_{1} P_{1}+n_{12} P_{2}-n_{21} P_{1}, \\
& \dot{P_{2}}=-\mu_{2} P_{2}+a e Q_{2} P_{2}-n_{12} P_{2}+n_{21} P_{1} .
\end{aligned}
$$



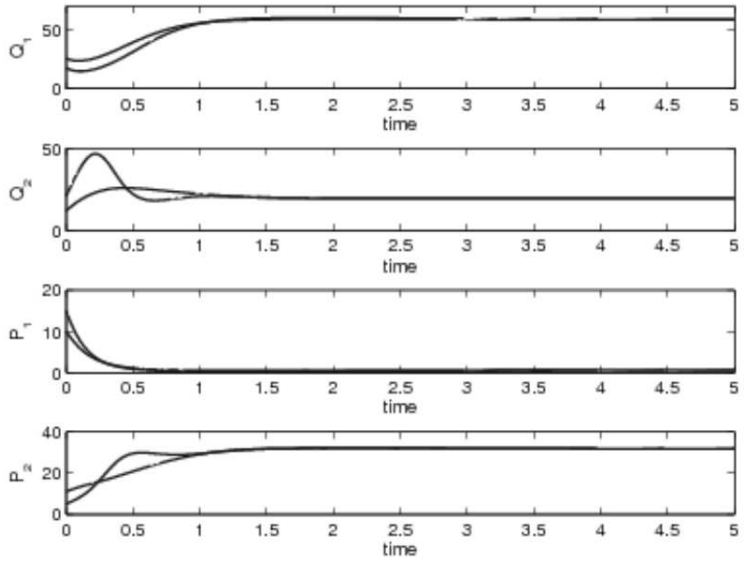

Figure 6: Equilibrium $X^{(4)}$ with no predators in patch 1, i.e. $P_{1}=0$, for the parameter values $r=8$, $k_{1}=100, k_{2}=150, a=0.5, m_{12}=0, m_{21}=3, \mu_{1}=6, \mu_{2}=3, e=0.3, n_{12}=0, n_{21}=2.8$.
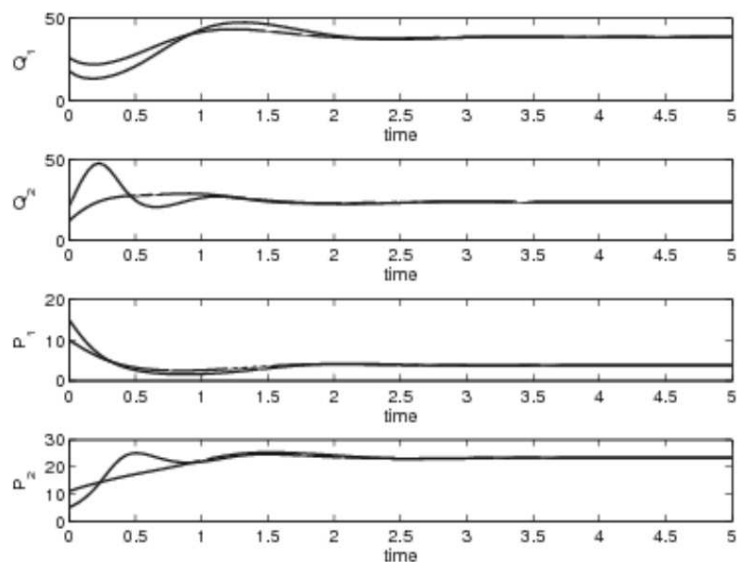

Figure 7: Coexistence equilibrium $X^{(5)}$, obtained for $r=8, k_{1}=100, k_{2}=150, a=0.5, m_{12}=0, m_{21}=3$, $\mu_{1}=3, \mu_{2}=4, e=0.3, n_{12}=0, n_{21}=2.8$.

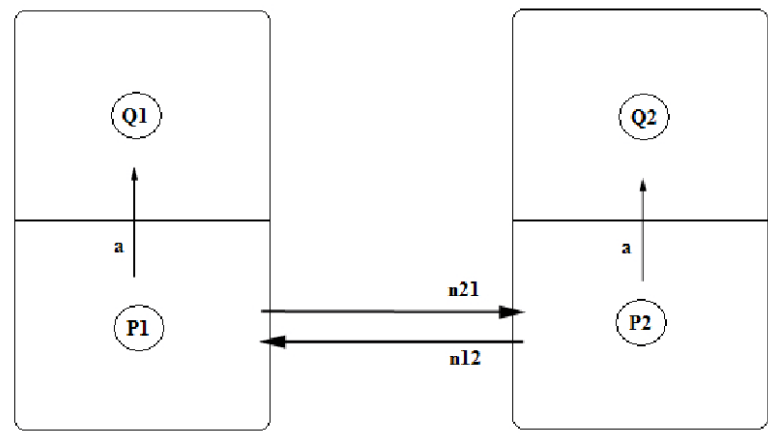

Figure 8: The model (4.1). 


\subsection{Equilibria}

The model (4.1) has the following equilibria: the origin $Y^{(0)}$; the readily established points $Y^{(1)}=\left(0, k_{2}, 0,0\right), Y^{(3)}=\left(k_{1}, 0,0,0\right)$ and $Y^{(5)}=\left(k_{1}, k_{2}, 0,0\right)$; the points $Y^{(2)}=$ $\left(0, Q_{2}^{(2)}, P_{1}^{(2)}, P_{2}^{(2)}\right)$ and $Y^{(4)}=\left(Q_{1}^{(4)}, 0, P_{1}^{(4)}, P_{2}^{(4)}\right)$; and finally the coexistence equilibrium $\mathscr{Y}^{(6)}$. The components of the points $Y^{(2)}=\left(0, Q_{2}^{(2)}, P_{1}^{(2)}, P_{2}^{(2)}\right)$ and $Y^{(4)}=\left(Q_{1}^{(4)}, 0, P_{1}^{(4)}, P_{2}^{(4)}\right)$ are given by

$$
\begin{aligned}
Q_{2}^{(2)} & =\frac{\sigma}{a e\left(\mu_{1}+n_{21}\right)}, & P_{1}^{(2)} & =\frac{r n_{12}\left[a e k_{2}\left(\mu_{1}+n_{21}\right)-\sigma\right]}{a^{2} e k_{2}\left(\mu_{1}+n_{21}\right)^{2}}, \\
P_{2}^{(2)} & =\frac{r\left[a e k_{2}\left(\mu_{1}+n_{21}\right)-\sigma\right]}{a^{2} e k_{2}\left(\mu_{1}+n_{21}\right)}, & Q_{1}^{(4)} & =\frac{\sigma}{a e\left(\mu_{2}+n_{12}\right)}, \\
P_{1}^{(4)} & =\frac{r\left[a e k_{1}\left(\mu_{2}+n_{12}\right)-\sigma\right]}{a^{2} e k_{1}\left(\mu_{2}+n_{12}\right)}, & P_{2}^{(4)} & =\frac{r n_{21}\left[a e k_{1}\left(\mu_{2}+n_{12}\right)-\sigma\right]}{a^{2} e k_{1}\left(\mu_{2}+n_{12}\right)^{2}} .
\end{aligned}
$$

Obviously, $Y^{(2)}$ is feasible if

$$
\operatorname{aek}_{2}\left(\mu_{1}+n_{21}\right)>\sigma
$$

and $Y^{(4)}$ is feasible if

$$
\operatorname{aek}_{1}\left(\mu_{2}+n_{12}\right)>\sigma \text {. }
$$

To find the coexistence equilibrium, we proceed as follows. From the first two equilibrium equations we find

$$
P_{1}^{(6)}=\frac{r}{a}\left(1-\frac{Q_{1}^{(6)}}{k_{1}}\right), \quad P_{2}^{(6)}=\frac{r}{a}\left(1-\frac{Q_{2}^{(6)}}{k_{2}}\right),
$$

which provide the first set of feasibility conditions

$$
Q_{1}^{(6)}<k_{1}, \quad Q_{2}^{(6)}<k_{2} .
$$

Letting

$$
\begin{aligned}
& A=\frac{a e}{k_{1}}>0, \quad B=\frac{\mu_{1}}{k_{1}}+\frac{n_{21}}{k_{1}}+a e>0, \quad C=\frac{n_{12}}{k_{2}}>0, \quad D=\mu_{1}+n_{21}-n_{12}, \\
& E=\frac{a e}{k_{2}}>0, \quad F=\frac{\mu_{2}}{k_{2}}+\frac{n_{12}}{k_{2}}+a e>0, \quad G=\frac{n_{21}}{k_{1}}>0, \quad H=\mu_{2}-n_{21}+n_{12},
\end{aligned}
$$

the last two equations can be rewritten as

$$
\psi_{1}: A Q_{1}^{2}-B Q_{1}+C Q_{2}+D=0 \text { and } \psi_{2}: E Q_{2}^{2}-F Q_{2}+G Q_{1}+H=0 .
$$

Evidently, we require the intersections of these two parabolae in the first quadrant. Now $\psi_{1}$ is concave, the vertex is $V_{1}=\left(B /(2 A),\left(B^{2}-4 A D\right) /(4 A C)\right)$ and it crosses the horizontal axis at $\left(B \pm \sqrt{B^{2}-4 A D}\right) /(2 A)$. Further, we readily find

$$
B^{2}-4 A D=\left(a e-\frac{\mu_{1}}{k_{1}}-\frac{n_{21}}{k_{1}}\right)^{2}+4 a e \frac{n_{12}}{k_{1}}>0
$$


such that $V_{1}$ lies in the first quadrant and one root is always positive. The parabola $\psi_{1}$ therefore has both positive roots if and only if $B-\sqrt{B^{2}-4 A D}>0$, which is equivalent to

$$
D>0 \text {. }
$$

The parabola $\psi_{2}$ has vertex $V_{2}=\left(\left(F^{2}-4 E H\right) /(4 E G), F /(2 E)\right)$ and crosses the vertical axis at $\left(F \pm \sqrt{F^{2}-4 E H}\right) /(2 E)$. Again we find $F^{2}-4 E H>0$ such that $V_{2}$ lies in the first quadrant and one of the two intersections is always positive. Moreover, both are positive if and only if

$$
H>0 \text {. }
$$

We can classify the possible situations arising in this context as follows:

- $V_{1}$ lies above a branch of the parabola $\psi_{2}$ if

$$
\frac{B^{2}-4 A D}{2 A C} \geq \frac{1}{E}\left[F-\sqrt{F^{2}-4 E\left(\frac{G B}{2 A}+H\right)}\right] ;
$$

- $V_{1}$ is above both branches of the parabola $\psi_{2}$ if

$$
\frac{B^{2}-4 A D}{2 A C} \geq \frac{1}{E}\left[F+\sqrt{F^{2}-4 E\left(\frac{G B}{2 A}+H\right)}\right] ;
$$

- $V_{2}$ lies to the right of one branch of the parabola $\psi_{1}$ if

$$
\frac{F^{2}-4 E H}{2 E G} \geq \frac{1}{A}\left[B-\sqrt{B^{2}-4 A\left(\frac{C F}{2 E}+D\right)}\right] ;
$$

- $V_{2}$ is located to the right of both branches of the parabola $\psi_{1}$ if

$$
\frac{F^{2}-4 E H}{2 E G} \geq \frac{1}{A}\left[B+\sqrt{B^{2}-4 A\left(\frac{C F}{2 E}+D\right)}\right] .
$$

Thus the following cases may arise, as depicted in Figs. 9-11:

- if (4.8) and (4.10) hold there is at least one intersection;

- if (4.5), (4.8) and (4.10) hold there are at least two intersections ;

- if (4.6), (4.8) and (4.10) hold there are at least two intersections ;

- if (4.5), (4.6), (4.8) and (4.10) hold there are four intersections ;

- if (4.5) and (4.9) hold there is at least one intersection ; and

- if (4.6) and (4.7) hold there is at least one intersection .

Of these, those that are feasible lie in the box $\left(0, k_{1}\right) \times\left(0, k_{2}\right)$. Since the coexistence equilibrium may not be unique, we denote it by a family of points

$$
\begin{aligned}
\mathscr{Y}^{(6)} & =\left\{\left(Q_{1}^{(6)}, Q_{2}^{(6)}, P_{1}^{(6)}, P_{2}^{(6)}\right)\right\} \\
& =\left\{\left(Q_{1}^{(6)}, Q_{2}^{(6)}, \frac{r}{a}\left(1-\frac{Q_{1}^{(6)}}{k_{1}}\right), \frac{r}{a}\left(1-\frac{Q_{2}^{(6)}}{k_{2}}\right)\right)\right\} .
\end{aligned}
$$



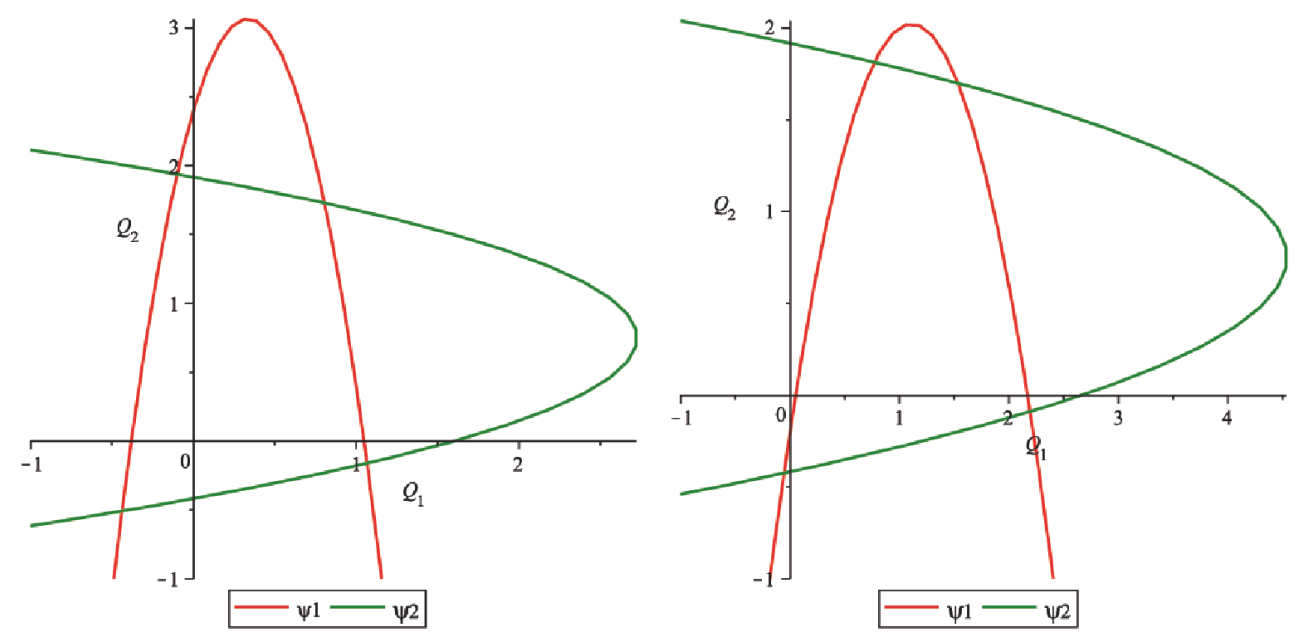

Figure 9: Left: The two parabolae for the parameter values $A=3, B=2, C=0.5, D=-1.2, E=1, F=$ $1.5, G=0.5, H=-0.8$ have at least one intersection in the first quadrant. Right: The two parabolae for the parameter values $A=0.9, B=2, C=0.5, D=0.1, E=1, F=1.5, G=0.3, H=-0.8$ have at least two intersections in the first quadrant.

\subsection{Stability}

The Jacobian of the system is

$$
J=\left(\begin{array}{cccc}
J_{11} & 0 & -a Q_{1} & 0 \\
0 & J_{22} & 0 & -a Q_{2} \\
a e P_{1} & 0 & J_{33} & n_{12} \\
0 & a e P_{2} & n_{21} & J_{44}
\end{array}\right)
$$

with

$$
\begin{array}{ll}
J_{11}=r\left(1-2 \frac{Q_{1}}{k_{1}}\right)-a P_{1}, & J_{22}=r\left(1-2 \frac{Q_{2}}{k_{2}}\right)-a P_{2}, \\
J_{33}=-\mu_{1}+a e Q_{1}-n_{21}, & J_{44}=-\mu_{2}+a e Q_{2}-n_{12} .
\end{array}
$$

The origin $Y^{(0)}$ is unconditionally unstable, given the two eigenvalues $\lambda_{1}=\lambda_{2}=r>0$. Similarly, at $Y^{(1)}$ two of the eigenvalues are $\lambda_{1}=-r$ and $\lambda_{2}=r>0$, implying instability; and at $Y^{(3)}$ we have $\lambda=r>0$, implying unconditional instability. At $Y^{(4)}$, one eigenvalue $\lambda_{1}=r-a P_{2}^{(4)}$ factors out, yielding the stability condition

$$
k_{1} a e\left(\mu_{2}+n_{12}\right)^{2}<n_{21}\left[k_{1} a e\left(\mu_{2}+n_{12}\right)-\sigma\right] .
$$

The remaining eigenvalues are the roots of the cubic

$$
\begin{aligned}
& \lambda^{3}-\left(J_{11}^{(4)}+J_{33}^{(4)}+J_{44}^{(4)}\right) \lambda^{2}+\left(J_{11}^{(4)} J_{44}^{(4)}+J_{11}^{(4)} J_{33}^{(4)}+J_{33}^{(4)} J_{44}^{(4)}-n_{12} n_{21}+a^{2} e Q_{1}^{(4)} P_{1}^{(4)}\right) \lambda \\
& -J_{11}^{(4)} J_{33}^{(4)} J_{44}^{(4)}+n_{12} n_{21} J_{11}^{(4)}-a^{2} e Q_{1}^{(4)} P_{1}^{(4)} J_{44}^{(4)}=0,
\end{aligned}
$$



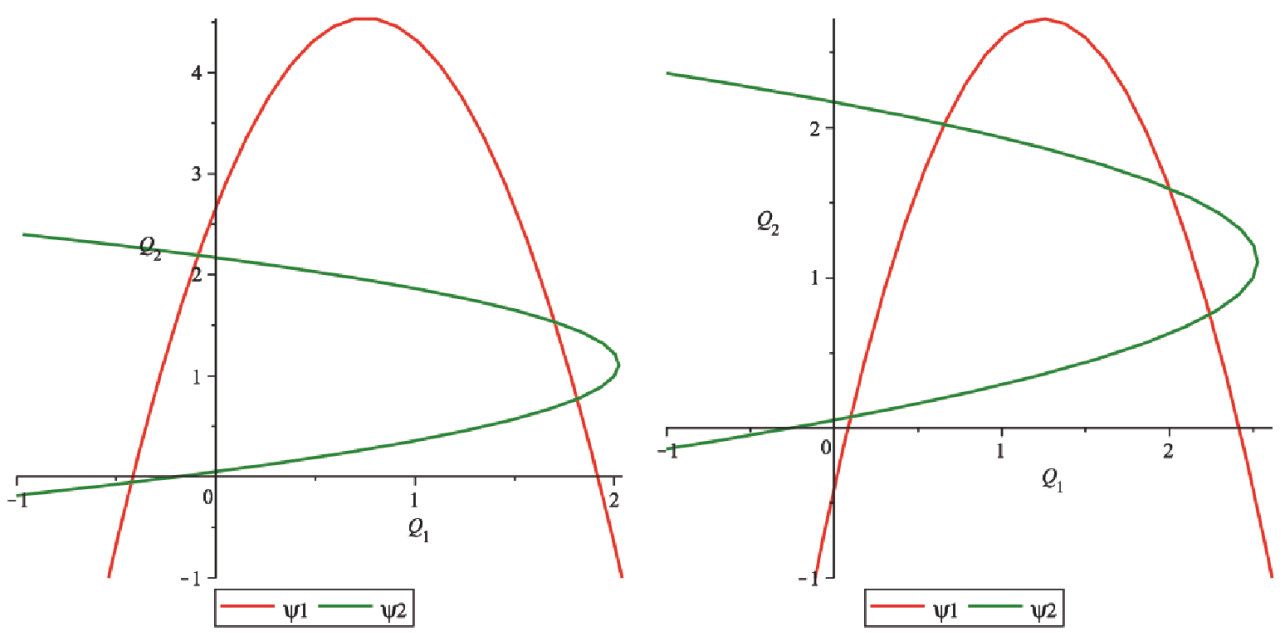

Figure 10: Left: The two parabolae for the parameter values $A=1, B=1.5, C=0.3, D=-0.8, E=$ $0.9, F=2, G=0.5, H=0.1$ have at least two intersections in the first quadrant. Right: The two parabolae for the parameter values $A=1, B=2.5, C=0.5, D=0.2, E=0.9, F=2, G=0.4, H=0.1$ have four intersections in the first quadrant.
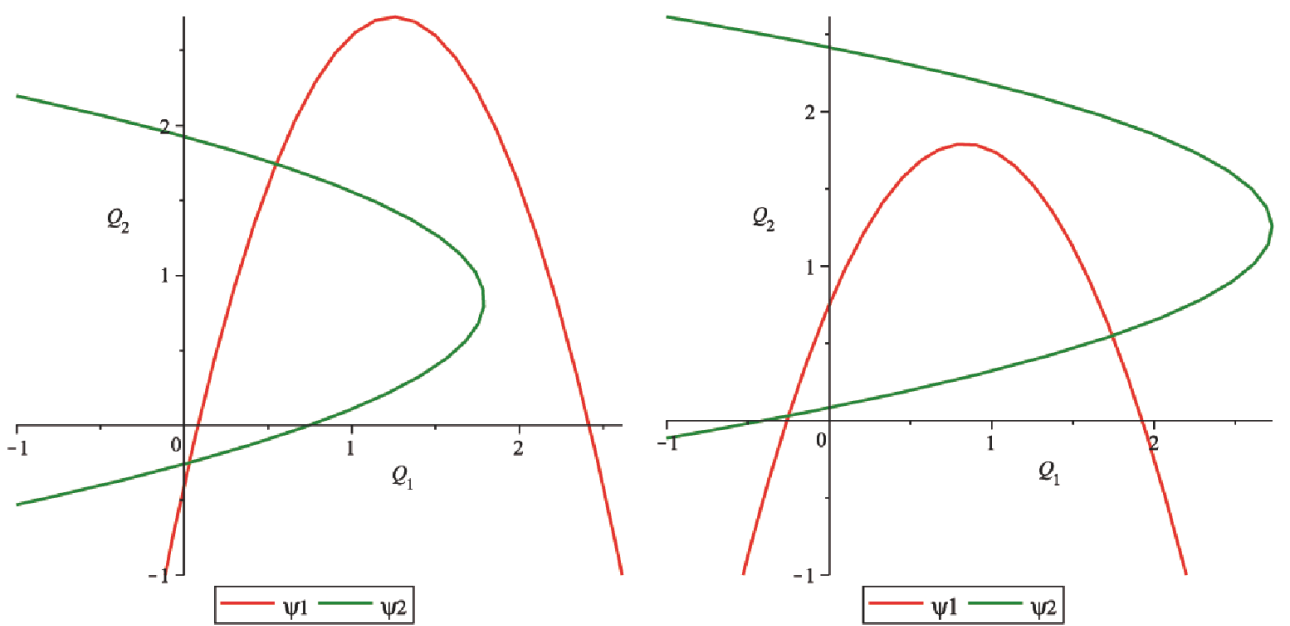

Figure 11: Left: The two parabolae for the parameter values $A=1, B=2.5, C=0.5, D=0.2, E=1.2, F=$ $2, G=0.8, H=-0.6$ have at least one intersection in the first quadrant. Right: The two parabolae for the parameter values $A=1.2, B=2, C=0.8, D=-0.6, E=1, F=2.5, G=0.5, H=0.2$ have at least one intersection in the first quadrant.

for which the Routh-Hurwitz conditions are always satisfied. The first is

$$
-J_{11}^{(4)} J_{33}^{(4)} J_{44}^{(4)}+n_{12} n_{21} J_{11}^{(4)}-a^{2} e Q_{1}^{(4)} P_{1}^{(4)} J_{44}^{(4)}>0
$$

that reduces to

$$
\sigma<k_{1} a e\left(\mu_{2}+n_{12}\right) \text {, }
$$

and from inequality (4.3) is satisfied. For the remaining two, we have

$$
-J_{11}^{(4)}-J_{33}^{(4)}-J_{44}^{(4)}=\left[\left(\mu_{1}+n_{21}\right)^{2}+n_{12} n_{21}\right] k_{1} a e+r \sigma>0
$$


and

$$
\begin{aligned}
& \left(-J_{11}^{(4)}-J_{33}^{(4)}-J_{44}^{(4)}\right)\left(J_{11}^{(4)} J_{44}^{(4)}+J_{11}^{(4)} J_{33}^{(4)}+J_{33}^{(4)} J_{44}^{(4)}-n_{12} n_{21}+a^{2} e Q_{1}^{(4)} P_{1}^{(4)}\right) \\
& >-J_{11}^{(4)} J_{33}^{(4)} J_{44}^{(4)}+n_{12} n_{21} J_{11}^{(4)}-a^{2} e Q_{1}^{(4)} P_{1}^{(4)} J_{44}^{(4)}
\end{aligned}
$$

that becomes

$$
\begin{aligned}
& {\left[k_{1} a e\left(\mu_{2}+n_{12}\right)-\sigma\right]\left(\frac{r \sigma}{k_{1} a e}+n_{12} n_{21}\right)+\left[\left(\mu_{2}+n_{12}\right)^{2}+n_{12} n_{21}\right]^{2}} \\
& +\frac{r \sigma}{k_{1} a e}\left[\left(\mu_{2}+n_{12}\right)^{2}+n_{12} n_{21}\right]>0
\end{aligned}
$$

which is again satisfied from inequality (4.3). Thus the stability of $Y^{(4)}$ depends only on inequality (4.11). Similarly, the stability of $Y^{(2)}$ only depends on the condition

$$
k_{2} a e\left(\mu_{1}+n_{21}\right)^{2}<n_{12}\left[k_{2} a e\left(\mu_{1}+n_{21}\right)-\sigma\right] .
$$

For the equilibrium $Y^{(5)}$, two eigenvalues are $\lambda_{1}=\lambda_{2}=-r<0$ and the others are the roots of a quadratic, for which the Routh-Hurwitz stability conditions become

$$
\begin{aligned}
& \operatorname{ae}\left(k_{1}+k_{2}\right)<\mu_{1}+\mu_{2}+n_{12}+n_{21}, \\
& \operatorname{aek}_{1}\left(\mu_{2}+n_{12}\right)+a e k_{2}\left(\mu_{1}+n_{21}\right)<\sigma+a^{2} e^{2} k_{1} k_{2} .
\end{aligned}
$$

At $\mathscr{Y}^{(6)}$, the characteristic equation is $\sum_{i=0}^{4} \lambda^{i} a_{i}=0$ where

$$
\begin{aligned}
a_{3}= & -J_{11}^{(6)}-J_{22}^{(6)}-J_{33}^{(6)}-J_{44}^{(6)} \\
a_{2}= & J_{22}^{(6)} J_{44}^{(6)}+\left(J_{22}^{(6)}+J_{44}^{(6)}\right)\left(J_{11}^{(6)}+J_{33}^{(6)}\right)+J_{11}^{(6)} J_{33}^{(6)}+a^{2} e\left(P_{1}^{(6)} Q_{1}^{(6)}+P_{2}^{(6)} Q_{2}^{(6)}\right)-n_{12} n_{21}, \\
a_{1}= & -J_{22}^{(6)} J_{44}^{(6)}\left(J_{11}^{(6)}+J_{33}^{(6)}\right)-J_{11}^{(6)} J_{33}^{(6)}\left(J_{22}^{(6)}+J_{44}^{(6)}\right)-a^{2} e P_{1}^{(6)} Q_{1}^{(6)}\left(J_{22}^{(6)}+J_{44}^{(6)}\right) \\
& -a^{2} e P_{2}^{(6)} Q_{2}^{(6)}\left(J_{44}^{(6)}+J_{33}^{(6)}\right)+n_{12} n_{21}\left(J_{11}^{(6)}+J_{22}^{(6)}\right) \\
a_{0}= & J_{11}^{(6)} J_{22}^{(6)} J_{33}^{(6)} J_{44}^{(6)}+a^{2} e P_{1}^{(6)} Q_{1}^{(6)} J_{22}^{(6)} J_{44}^{(6)}+a^{2} e P_{2}^{(6)} Q_{2}^{(6)} J_{11}^{(6)} J_{33}^{(6)}+a^{4} e^{2} P_{1}^{(6)} P_{2}^{(6)} Q_{1}^{(6)} Q_{2}^{(6)} \\
& -n_{12} n_{21} J_{11}^{(6)} J_{22}^{(6)} .
\end{aligned}
$$

The Routh-Hurwitz conditions are

$$
\begin{aligned}
& a e\left(Q_{1}^{(6)}+Q_{2}^{(6)}\right)<r\left(\frac{Q_{1}^{(6)}}{k_{1}}+\frac{Q_{2}^{(6)}}{k_{2}}\right)+\mu_{1}+\mu_{2}+n_{12}+n_{21} \\
& \sigma+a e\left(4 a e Q_{1}^{(6)} Q_{2}^{(6)}+\mu_{2} k_{1}+\mu_{1} k_{2}+n_{12} k_{1}+n_{21} k_{2}+a e k_{1} k_{2}\right)>2 a e\left(\mu_{1} Q_{2}^{(6)}+\mu_{2} Q_{1}^{(6)}\right. \\
& \left.\quad+n_{12} Q_{1}^{(6)}+n_{21} Q_{2}^{(6)}+a e k_{1} Q_{2}^{(6)}+a e k_{2} Q_{1}^{(6)}+a e k_{1} \frac{Q_{2}^{(6)}}{k_{2}}+a e k_{2} \frac{Q_{1}^{(6)}}{k_{1}}\right)
\end{aligned}
$$




$$
\begin{aligned}
& {\left[J_{22}^{(6)} J_{44}^{(6)}+\left(J_{22}^{(6)}+J_{44}^{(6)}\right)\left(J_{11}^{(6)}+J_{33}^{(6)}\right)+J_{11}^{(6)} J_{33}^{(6)}+a^{2} e\left(P_{1}^{(6)} Q_{1}^{(6)}+P_{2}^{(6)} Q_{2}^{(6)}\right)-n_{12} n_{21}\right]} \\
& \quad \times\left(-J_{11}^{(6)}-J_{22}^{(6)}-J_{33}^{(6)}-J_{44}^{(6)}\right)>-J_{22}^{(6)} J_{44}^{(6)}\left(J_{11}^{(6)}+J_{33}^{(6)}\right)-J_{11}^{(6)} J_{33}^{(6)}\left(J_{22}^{(6)}+J_{44}^{(6)}\right) \\
& \quad-a^{2} e P_{1}^{(6)} Q_{1}^{(6)}\left(J_{22}^{(6)}+J_{44}^{(6)}\right)-a^{2} e P_{2}^{(6)} Q_{2}^{(6)}\left(J_{44}^{(6)}+J_{33}^{(6)}\right)+n_{12} n_{21}\left(J_{11}^{(6)}+J_{22}^{(6)}\right), \\
& {\left[-J_{22}^{(6)} J_{44}^{(6)}\left(J_{11}^{(6)}+J_{33}^{(6)}\right)-J_{11}^{(6)} J_{33}^{(6)}\left(J_{22}^{(6)}+J_{44}^{(6)}\right)-a^{2} e P_{1}^{(6)} Q_{1}^{(6)}\left(J_{22}^{(6)}+J_{44}^{(6)}\right)\right.} \\
& \left.\quad-a^{2} e P_{2}^{(6)} Q_{2}^{(6)}\left(J_{44}^{(6)}+J_{33}^{(6)}\right)+n_{12} n_{21}\left(J_{11}^{(6)}+J_{22}^{(6)}\right)\right]\left\{\left[J_{22}^{(6)} J_{44}^{(6)}+\left(J_{22}^{(6)}+J_{44}^{(6)}\right)\left(J_{11}^{(6)}+J_{33}^{(6)}\right)\right.\right. \\
& \left.\quad+J_{11}^{(6)} J_{33}^{(6)}+a^{2} e\left(P_{1}^{(6)} Q_{1}^{(6)}+P_{2}^{(6)} Q_{2}^{(6)}\right)-n_{12} n_{21}\right]\left(-J_{11}^{(6)}-J_{22}^{(6)}-J_{33}^{(6)}-J_{44}^{(6)}\right) \\
& \quad-\left[-J_{22}^{(6)} J_{44}^{(6)}\left(J_{11}^{(6)}+J_{33}^{(6)}\right)-J_{11}^{(6)} J_{33}^{(6)}\left(J_{22}^{(6)}+J_{44}^{(6)}\right)-a^{2} e P_{1}^{(6)} Q_{1}^{(6)}\left(J_{22}^{(6)}+J_{44}^{(6)}\right)\right. \\
& \left.\left.\quad-a^{2} e P_{2}^{(6)} Q_{2}^{(6)}\left(J_{44}^{(6)}+J_{33}^{(6)}\right)+n_{12} n_{21}\left(J_{11}^{(6)}+J_{22}^{(6)}\right)\right]\right\}>\left[J_{11}^{(6)} J_{22}^{(6)} J_{33}^{(6)} J_{44}^{(6)}+a^{2} e P_{1}^{(6)} Q_{1}^{(6)} J_{22}^{(6)} J_{44}^{(6)}\right. \\
& \left.\quad+a^{2} e P_{2}^{(6)} Q_{2}^{(6)} J_{11}^{(6)} J_{33}^{(6)}+a^{4} e^{2} P_{1}^{(6)} P_{2}^{(6)} Q_{1}^{(6)} Q_{2}^{(6)}-n_{12} n_{21} J_{11}^{(6)} J_{22}^{(6)}\right] \\
& \quad \times\left(-J_{11}^{(6)}-J_{22}^{(6)}-J_{33}^{(6)}-J_{44}^{(6)}\right)^{2} .
\end{aligned}
$$

In Fig. 12 we show that (4.13) do not constitute an empty set so the predator-free equilibrium $Y^{(5)}$ is stable, using the parameters $r=8, k_{1}=100, k_{2}=150, a=0.5, m_{12}=0$, $m_{21}=0, \mu_{1}=7, \mu_{2}=8, e=0.1, n_{12}=2.5$ and $n_{21}=2.8$. We also verified that the coexistence equilibrium $\mathscr{Y}^{(6)}$ can be achieved - cf. Fig. 13 for the parameter values $r=8$, $k_{1}=100, k_{2}=150, a=0.5, m_{12}=0, m_{21}=0, \mu_{1}=3, \mu_{2}=4, e=0.3, n_{12}=2.5$, $n_{21}=2.8$.

\section{Only Prey Migrate}

The scheme when only prey migrate is represented in Fig. 14, and corresponds to setting $n_{12}=n_{21}=0$ in (2.1). Thus the model becomes

$$
\begin{aligned}
& \dot{Q_{1}}=r Q_{1}\left(1-\frac{Q_{1}}{k_{1}}\right)-a Q_{1} P_{1}+m_{12} Q_{2}-m_{21} Q_{1}, \\
& \dot{Q_{2}}=r Q_{2}\left(1-\frac{Q_{2}}{k_{2}}\right)-a Q_{2} P_{2}-m_{12} Q_{2}+m_{21} Q_{1}, \\
& \dot{P_{1}}=-\mu_{1} P_{1}+a e Q_{1} P_{1}, \\
& \dot{P_{2}}=-\mu_{2} P_{2}+a e Q_{2} P_{2} .
\end{aligned}
$$

\subsection{Equilibria}

There now are only five possible equilibria: the origin $Z^{(0)}$, the boundary equilibria $Z^{(1)}=\left(Q_{1}^{(1)}, Q_{2}^{(1)}, 0,0\right), Z^{(2)}=\left(Q_{1}^{(2)}, Q_{2}^{(2)}, 0, P_{2}^{(2)}\right)$ and $Z^{(3)}=\left(Q_{1}^{(3)}, Q_{2}^{(3)}, P_{1}^{(3)}, 0\right)$, and coexistence, $Z^{(4)}=\left(Q_{1}^{(4)}, Q_{2}^{(4)}, P_{1}^{(4)}, P_{2}^{(4)}\right)$. We thus find one point discovered earlier - viz. 

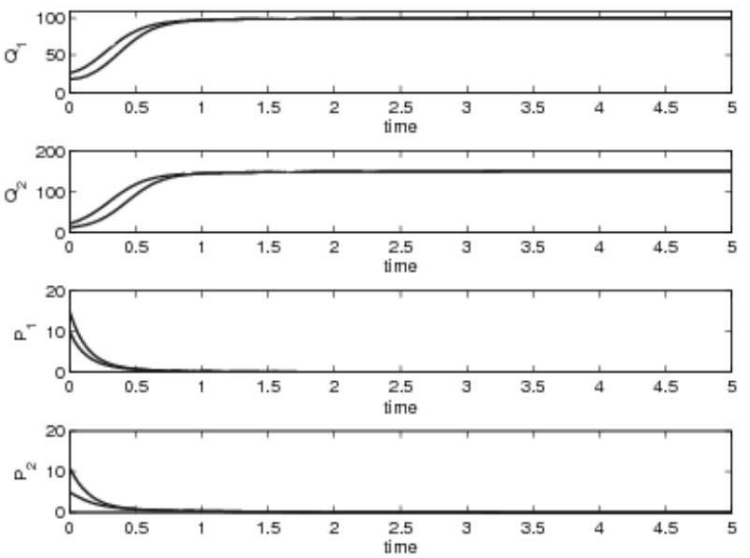

Figure 12: Predator-free equilibrium $Y^{(5)}$ obtained for the parameter values $r=8, k_{1}=100, k_{2}=150$, $a=0.5, m_{12}=0, m_{21}=0, \mu_{1}=7, \mu_{2}=8, e=0.1, n_{12}=2.5, n_{21}=2.8$.
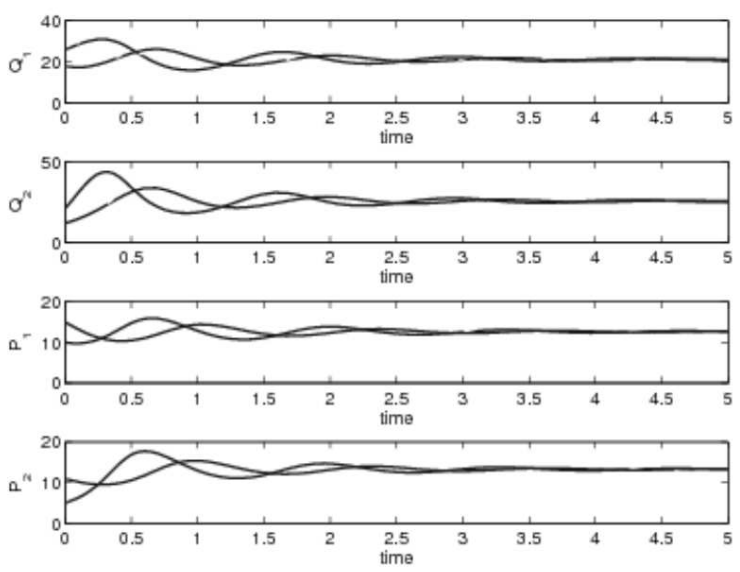

Figure 13: Coexistence equilibrium $\mathscr{Y}^{(6)}$ for the parameter values $r=8, k_{1}=100, k_{2}=150, a=0.5$, $m_{12}=0, m_{21}=0, \mu_{1}=3, \mu_{2}=4, e=0.3, n_{12}=2.5, n_{21}=2.8$.

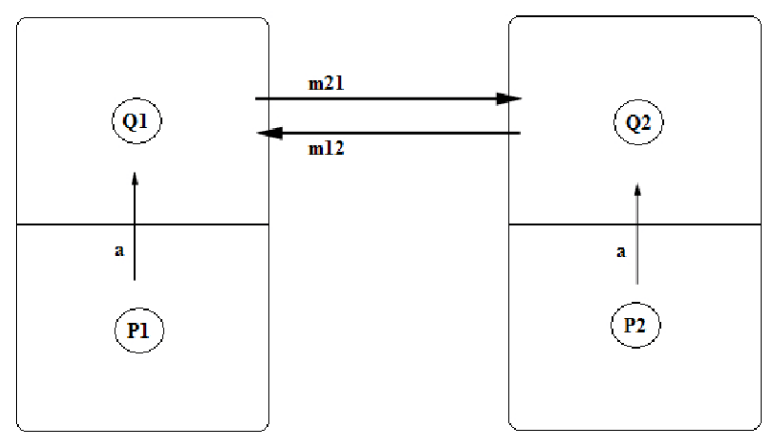

Figure 14: Grafical representation of the model (5.1). 
$Z^{(1)} \equiv E^{(1)}$, which is unconditionally feasible. We also obtain

$$
\begin{aligned}
& Q_{1}^{(2)}=\frac{k_{1}}{2 r}\left[r-m_{21}+\sqrt{\left(r-m_{21}\right)^{2}+4 m_{12} \frac{r \mu_{2}}{k_{1} a e}}\right], \quad Q_{2}^{(2)}=\frac{\mu_{2}}{a e}, \\
& P_{2}^{(2)}=\frac{r}{a}\left(1-\frac{\mu_{2}}{a e k_{2}}\right)-\frac{m_{12}}{a}+\frac{m_{21} k_{1} e}{2 r \mu_{2}}\left(r-m_{21}+\sqrt{\left(r-m_{21}\right)^{2}+4 m_{12} \frac{r \mu_{2}}{k_{1} a e}}\right),
\end{aligned}
$$

so $Z^{(2)}=\left(Q_{1}^{(2)}, Q_{2}^{(2)}, 0, P_{2}^{(2)}\right)$, is feasible for

$$
r+\frac{m_{21} k_{1} a e}{2 r \mu_{2}}\left(r-m_{21}+\sqrt{\left(r-m_{21}\right)^{2}+4 m_{12} \frac{r \mu_{2}}{k_{1} a e}}\right)>\frac{r \mu_{2}}{a e k_{2}}+m_{12} .
$$

For $Z^{(3)}=\left(Q_{1}^{(3)}, Q_{2}^{(3)}, P_{1}^{(3)}, 0\right)$, we have

$$
\begin{aligned}
& Q_{1}^{(3)}=\frac{\mu_{1}}{a e}, \quad Q_{2}^{(3)}=\frac{k_{2}}{2 r}\left[r-m_{12}+\sqrt{\left(r-m_{12}\right)^{2}+4 m_{21} \frac{r \mu_{1}}{k_{2} a e}}\right], \\
& P_{1}^{(3)}=\frac{r}{a}\left(1-\frac{r \mu_{1}}{k_{1} a e}\right)-\frac{m_{21}}{a}+\frac{m_{12} k_{2} e}{2 r \mu_{1}}\left(r-m_{12}+\sqrt{\left(r-m_{12}\right)^{2}+4 m_{21} \frac{r \mu_{1}}{k_{2} a e}}\right),
\end{aligned}
$$

so it is feasible for

$$
r+\frac{m_{12} k_{2} a e}{2 \mu_{1}}\left(r-m_{12}+\sqrt{\left(r-m_{12}\right)^{2}+4 m_{21} \frac{r \mu_{1}}{k_{2} a e}}\right)>\frac{r \mu_{1}}{a e k_{1}}+m_{21} .
$$

For the coexistence case, the equilibrium can once again be evaluated explicitly. Thus

$$
\begin{array}{ll}
Q_{1}^{(4)}=\frac{\mu_{1}}{a e}, & P_{1}^{(4)}=\frac{1}{a}\left[r\left(1-\frac{\mu_{1}}{k_{1} a e}\right)+m_{12} \frac{\mu_{2}}{\mu_{1}}-m_{21}\right], \\
Q_{2}^{(4)}=\frac{\mu_{2}}{a e}, & P_{2}^{(4)}=\frac{1}{a}\left[r\left(1-\frac{\mu_{2}}{k_{2} a e}\right)+m_{21} \frac{\mu_{1}}{\mu_{2}}-m_{12}\right],
\end{array}
$$

so the coexistence equilibrium is feasible if

$$
r+m_{12} \frac{\mu_{2}}{\mu_{1}}>\frac{r \mu_{1}}{k_{1} a e}+m_{21}, \quad r+m_{21} \frac{\mu_{1}}{\mu_{2}}>\frac{r \mu_{2}}{k_{2} a e}+m_{12} .
$$

\subsection{Stability}

The Jacobian for model (5.1) is

$$
J=\left(\begin{array}{cccc}
J_{11} & m_{12} & -a Q_{1} & 0 \\
m_{21} & J_{22} & 0 & -a Q_{2} \\
a e P_{1} & 0 & -\mu_{1}+a e Q_{1} & 0 \\
0 & a e P_{2} & 0 & -\mu_{2}+a e Q_{2}
\end{array}\right),
$$


with

$$
J_{11}=r\left(1-2 \frac{Q_{1}}{k_{1}}\right)-a P_{1}-m_{21}, \quad J_{22}=r\left(1-2 \frac{Q_{2}}{k_{2}}\right)-a P_{2}-m_{12} .
$$

At $Z^{(0)}$, the eigenvalues are $\lambda_{1}=-\mu_{1}<0, \lambda_{2}=-\mu_{2}<0, \lambda_{3}=m_{12}+m_{21}-r$ and $\lambda_{4}=r>0$, so that the equilibrium is unconditionally unstable. At $Z^{(1)}$, two eigenvalues are $\lambda_{1}=a e Q_{2}^{(1)}-\mu_{2}$ and $\lambda_{2}=a e Q_{1}^{(1)}-\mu_{1}$ and the other two are the roots of the quadratic

$$
\left(r-\frac{2 r Q_{1}^{(1)}}{k_{1}}-m_{21}-\lambda\right)\left(r-\frac{2 r Q_{2}^{(1)}}{k_{2}}-m_{12}-\lambda\right)-m_{12} m_{21}=0 .
$$

From its Routh-Hurwitz conditions, and imposing negativity for the first two eigenvalues, we get the stability conditions

$$
\begin{aligned}
& \mu_{2}>a e Q_{2}^{(1)}, \quad \mu_{1}>a e Q_{1}^{(1)}, \quad 2 r<2 r\left(\frac{Q_{1}^{(1)}}{k_{1}}+\frac{Q_{2}^{(1)}}{k_{2}}\right)+m_{12}+m_{21}, \\
& r+\frac{4 r Q_{1}^{(1)} Q_{2}^{(1)}}{k_{1} k_{2}}+\frac{2 m_{12} Q_{1}^{(1)}}{k_{1}}+\frac{2 m_{21} Q_{2}^{(1)}}{k_{2}}>\frac{2 r Q_{1}^{(1)}}{k_{1}}+\frac{2 r Q_{2}^{(1)}}{k_{2}}+m_{21}+m_{12} .
\end{aligned}
$$

For $Z^{(2)}$, one eigenvalue is readily determined as $\lambda_{1}=-\mu_{1}+a e Q_{1}^{(2)}$, giving the first stability condition

$$
\mu_{1}>\frac{k_{1} a e}{2 r}\left[r-m_{21}+\sqrt{\left(r-m_{21}\right)^{2}+\frac{4 m_{12} r \mu_{2}}{k_{1} a e}}\right],
$$

and the remainder come from the roots of the cubic equation

$$
\lambda^{3}-\left(J_{11}^{(2)}+J_{22}^{(2)}\right) \lambda^{2}+\left(\mu_{2} a P_{2}^{(2)}+J_{11}^{(2)} J_{22}^{(2)}-m_{21} m_{12}\right) \lambda-\mu_{2} a P_{2}^{(2)} J_{11}^{(2)}=0 .
$$

Thus the Routh-Hurwitz conditions $J_{11}^{(2)}+J_{22}^{(2)}<0$ and $-\mu_{2} a P_{2}^{(2)} J_{11}^{(2)}>0$ give the first set of inequalities needed for stability

$$
2 r<\frac{2 r Q_{1}^{(2)}}{k_{1}}+\frac{2 r \mu_{2}}{k_{2} a e}+a P_{2}^{(2)}+m_{12}+m_{21}, \quad r<\frac{2 r Q_{1}^{(2)}}{k_{1}}+m_{21},
$$

while the fourth one $\left(J_{11}^{(2)}+J_{22}^{(2)}\right)\left(\mu_{2} a P_{2}^{(2)}+J_{11}^{(2)} J_{22}^{(2)}-m_{21} m_{12}\right)<\mu_{2} a P_{2}^{(2)} J_{11}^{(2)}$ provides the last stability condition

$$
\left(J_{11}^{(2)} J_{22}^{(2)}-m_{21} m_{12}\right)\left(J_{11}^{(2)}+J_{22}^{(2)}\right)+\mu_{2} a P_{2}^{(2)} J_{22}^{(2)}<0 .
$$

Thus $Z^{(2)}$ is stable when (5.6), (5.7), (5.8) are all satisfied. Similarly, for $Z^{(3)}$ we find the eigenvalue $\lambda_{1}=-\mu_{2}+a e Q_{2}^{(3)}$, for which the stability condition is

$$
\mu_{2}>\frac{k_{2} a e}{2 r}\left[r-m_{12}+\sqrt{\left(r-m_{12}\right)^{2}+4 m_{21} \frac{r \mu_{1}}{k_{2} a e}}\right] .
$$


The roots of

$$
\lambda^{3}-\left(J_{11}^{(3)}+J_{22}^{(3)}\right) \lambda^{2}+\left(\mu_{1} a P_{1}^{(3)}+J_{11}^{(3)} J_{22}^{(3)}-m_{21} m_{12}\right) \lambda-\mu_{1} a P_{1}^{(3)} J_{22}^{(3)}=0
$$

must have negative real parts. Once again, we conveniently use the associated RouthHurwitz conditions

$$
\begin{aligned}
& J_{11}^{(3)}+J_{22}^{(3)}<0, \quad-\mu_{1} a P_{1}^{(3)} J_{22}^{(3)}>0, \\
& \left(J_{11}^{(3)}+J_{22}^{(3)}\right)\left(\mu_{1} a P_{1}^{(3)}+J_{11}^{(3)} J_{22}^{(3)}-m_{21} m_{12}\right)<\mu_{1} a P_{1}^{(3)} J_{22}^{(3)},
\end{aligned}
$$

to obtain the remaining stability conditions

$$
\begin{aligned}
& 2 r<\frac{2 r Q_{2}^{(3)}}{k_{2}}+\frac{2 r \mu_{1}}{k_{1} a e}+a P_{1}^{(3)}+m_{12}+m_{21}, \quad r<\frac{2 r Q_{2}^{(3)}}{k_{2}}+m_{12}, \\
& \left(J_{11}^{(3)} J_{22}^{(3)}-m_{21} m_{12}\right)\left(J_{11}^{(3)}+J_{22}^{(3)}\right)+\mu_{1} a P_{1}^{(3)} J_{11}^{(3)}<0 .
\end{aligned}
$$

In summary, $Z^{(3)}$ is stable when both (5.9) and (5.10) are satisfied. At the coexistence equilibrium $Z^{(4)}$, we get the following fourth degree characteristic equation

$$
\begin{aligned}
& \lambda^{4}-\left(J_{11}^{(4)}+J_{22}^{(4)}\right) \lambda^{3}+\left(a \mu_{1} P_{1}^{(4)}+J_{11}^{(4)} J_{22}^{(4)}-m_{12} m_{21}+a \mu_{2} P_{2}^{(4)}\right) \lambda^{2} \\
& +\left(-a \mu_{1} P_{1}^{(4)} J_{22}^{(4)}-a \mu_{2} P_{2}^{(4)} J_{11}^{(4)}\right) \lambda+a^{2} \mu_{1} \mu_{2} P_{1}^{(4)} P_{2}^{(4)}=0 .
\end{aligned}
$$

One of the Routh-Hurwitz conditions holds unconditionally - viz. $a^{2} \mu_{1} \mu_{2} P_{1}^{(4)} P_{2}^{(4)}>0$. The remainder provide the stability conditions

$$
\begin{aligned}
& J_{11}^{(4)}+J_{22}^{(4)}<0, \\
& a \mu_{1} P_{1}^{(4)} J_{11}^{(4)}+a \mu_{2} P_{2}^{(4)} J_{22}^{(4)}+\left(J_{11}^{(4)} J_{22}^{(4)}-m_{12} m_{21}\right)\left(J_{11}^{(4)}+J_{22}^{(4)}\right)<0, \\
& \left(J_{11}^{(4)}+J_{22}^{(4)}\right)\left(\mu_{1} P_{1}^{(4)} J_{22}^{(4)}-\mu_{2} P_{2}^{(4)} J_{11}^{(4)}\right)\left(m_{12} m_{21}-J_{11}^{(4)} J_{22}^{(4)}\right) \\
& \quad<a J_{11}^{(4)} J_{22}^{(4)}\left(\mu_{1} P_{1}^{(4)}-\mu_{2} P_{2}^{(4)}\right)^{2} .
\end{aligned}
$$

Fig. 15 shows a simulation for $Z^{(2)}$, for the parameter values $r=10, k_{1}=100, k_{2}=150$, $a=0.5, m_{12}=2, m_{21}=3, \mu_{1}=12, \mu_{2}=3, e=0.2, n_{12}=0$ and $n_{21}=0$. Fig. 16 shows the coexistence equilibrium $Z^{(4)}$ for $r=8, k_{1}=100, k_{2}=150, a=0.5, m_{12}=2, m_{21}=3$, $\mu_{1}=3, \mu_{2}=4, e=0.3, n_{12}=0$ and $n_{21}=0$.

\section{Conclusions}

In the four metapopulation systems presented, the ecosystem can never disappear. This is a positive result from the ecological point of view, which may be ascribed to the model assumptions. Indeed, the prey are always assumed to have enough resources to thrive, so the eigenvalue $r$ at the origin is positive in all cases. Further, the prey always thrive in at least one patch, no matter what happens to the rest of the ecosystem. This agrees with the previous remark that the system cannot get extinguished - at least one population 

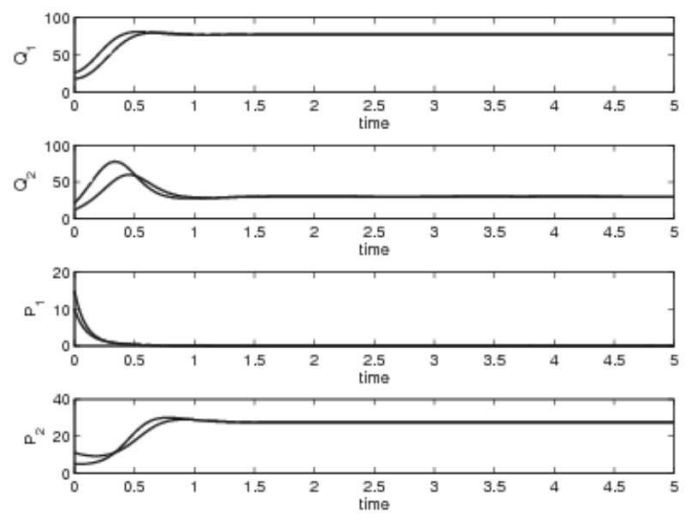

Figure 15: Equilibrium $Z^{(2)}$ with no predators in patch 1 obtained with $r=10, k_{1}=100, k_{2}=150$, $a=0.5, m_{12}=2, m_{21}=3, \mu_{1}=12, \mu_{2}=3, e=0.2, n_{12}=0, n_{21}=0$.
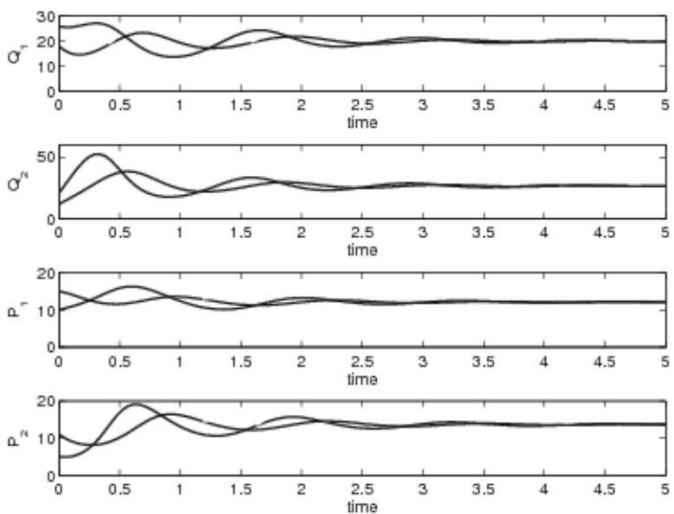

Figure 16: Coexistece equilibrium $Z^{(4)}$ obtained with $r=8, k_{1}=100, k_{2}=150, a=0.5, m_{12}=2, m_{21}=3$, $\mu_{1}=3, \mu_{2}=4, e=0.3, n_{12}=0, n_{21}=0$.

must survive, and that cannot be the predators alone (in the absence of their food source). If the whole ecosystem is wiped out except for a prey population in just one patch, the prey must thrive to carrying capacity. We summarise the possible system behaviour in the accompanying Table.

In the general two-patch model with migrations in both directions for both populations, there are only two possible steady states - viz. the predator-free equilibrium and the coexistence of all populations. There are restrictions for the existence of the predator-free equilibrium, but there is coexistence if the second prey population is bounded above, since the combination of (2.4) and (2.5) gives

$$
Q_{2}^{(2)} \leq \frac{1}{a e} \min \left\{\mu_{2}+n_{12}, \frac{\sigma}{\mu_{1}+n_{21}}\right\}
$$

together with a more complicated upper bound for $Q_{1}^{(2)}$, leading to three possibilities - 
Table 1: Summary of the possible system behaviour.

\begin{tabular}{l|llll}
\hline Model & Equilibrium & $\begin{array}{l}\text { Existence } \\
\text { condition }\end{array}$ & $\begin{array}{l}\text { Stability } \\
\text { condition }\end{array}$ & Interpretation \\
\hline General case & $E^{(0)}$ & - & unstable & system extinction \\
$(2.1)$ & $E^{(1)}$ & - & $(2.6),(2.7)$ & predator-free \\
& $E^{(2)}$ & $(2.4),(2.5)$ & $(2.8)-(2.11)$ & coexistence \\
\hline Migration & $X^{(0)}$ & - & unstable & system extinction \\
only from & $X^{(1)}$ & - & $(3.7)$ & only prey, in Patch 2 \\
Patch 1 & $X^{(2)}$ & $(3.2)$ & $(3.8)$ & Patch 1 empty \\
to & $X^{(3)}$ & $(3.3)$ & $(3.9)$ & predator-free \\
Patch 2 & $X^{(4)}$ & $(3.4)$ & $(3.10)$ & no predators in Patch 1 \\
(3.1) & $X^{(5)}$ & $(3.6)$ & $(3.11),(3.12)$ & coexistence \\
\hline Only & $Y^{(0)}$ & - & unstable & system extinction \\
predators & $Y^{(1)}$ & - & unstable & only prey, in Patch 2 \\
migrate & $Y^{(2)}$ & $(4.2)$ & $(4.12)$ & no prey in Patch 1 \\
(4.1) & $Y^{(3)}$ & - & unstable & only prey, in Patch 1 \\
& $Y^{(4)}$ & $(4.3)$ & $(4.11)$ & no prey in Patch 2 \\
& $Y^{(5)}$ & - & $(4.13)$ & predator-free \\
& $\mathscr{Y}^{(6)}$ & $(4.4)-(4.10)$ & $(4.14)-(4.17)$ & coexistence \\
\hline Only & $Z^{(0)}$ & - & unstable & system extinction \\
prey & $Z^{(1)}$ & - & $(5.5)$ & predator-free \\
migrate & $Z^{(2)}$ & $(5.2)$ & $(5.6)-(5.8)$ & no predators in Patch 1 \\
(5.1) & $Z^{(3)}$ & $(5.3)$ & $(5.9),(5.10)$ & no predators in Patch 2 \\
& $Z^{(4)}$ & $(5.4)$ & $(5.11)$ & coexistence \\
\hline
\end{tabular}

viz. no restriction if $m_{21} k_{2}>\operatorname{aer} Q_{2}^{(2)}$ and $m_{12}<r$; if $m_{21} k_{2}>\operatorname{aer} Q_{2}^{(2)}$ and $m_{12}>r$,

$$
Q_{1}^{(2)} \geq \frac{\operatorname{aek}_{2} Q_{2}^{(2)}}{m_{21} k_{2}-\operatorname{aer} Q_{2}^{(2)}}\left(m_{12}-r\right) ;
$$

and finally, if $m_{21} k_{2}<\operatorname{aer} Q_{2}^{(2)}$ and $m_{12}<r$ then

$$
Q_{1}^{(2)} \leq \frac{a e k_{2} Q_{2}^{(2)}}{m_{21} k_{2}-\operatorname{aer} Q_{2}^{(2)}}\left(m_{12}-r\right) .
$$

In some cases that we did not explore, the coexistence could become a multiple equilibrium. All these equilibria are found also in the remaining cases, with possibly different feasibility and stability conditions. However, the origin always retains its instability property, so the survival of at least part of the ecosystem is assured in all cases.

The model (3.1) with migrations only in one direction contains three more possible equilibria, in addition to those above. The additional outcomes are that the patch from 
which migrations are possible may empty (equilibrium $X^{(2)}$ ); or it contains only prey while the patch into which populations migrate is fully populated by both species (equilibrium $\left.X^{(4)}\right)$; or the prey thrive only in the second patch and the predators are completely wiped out (equilibrium $X^{(3)}$ ). The condition (3.2) for the feasibility of the patch 2 predator-prey coexistence equilibrium, $X^{(2)}$, says nothing about the properties of the patch from which the populations migrate, but requires the carrying capacity in the other environment to exceed the ratio between the predator mortality and their food uptake there - i.e.

$$
k_{2} \geq \frac{\mu_{2}}{a e} .
$$

Thus if the prey find a suitably rich environment in the second patch they tend to migrate there, leaving less resources for the predators in the first patch. The predators then tend to migrate to where the food is more abundant, and therefore abandon the first patch. When the patch is abandoned it cannot be repopulated, since migration back into it is forbidden. For stability, the prey reproduction rate must be lower than their immigrations from the other patch - cf. (3.8). However, this is the opposite condition for the feasibility of $X^{(4)}$, so a transcritical bifurcation occurs when (3.8) becomes an equality. Similarly, another transcritical bifurcation stems from the second condition in (3.4) and the second condition in (3.9), when the equilibria $X^{(3)}$ and $X^{(4)}$ collide - as well as from (3.6) and (3.10), for the coalescence of the equilibria $X^{(4)}$ and $X^{(5)}$. Further, both equilibria $X^{(1)}$ and $X^{(2)}$ entail the extinction of both populations in the patch where the migrations originate. This fact could be exploited, for instance when it is necessary to naturally displace a population in order to reclaim a ground.

In (4.1), when only predators are allowed to migrate, the most striking result is that prey do not thrive alone in either of the two patches, as the equilibria $Y^{(1)}$ and $Y^{(3)}$ are unstable. The reason again is that one eigenvalue is $r>0$. Thus prey reproduction available in the other patch prevents the first patch from remaining empty. The prey can disappear from either patch at equilibria $Y^{(2)}$ and $Y^{(4)}$, but then the predators survive in both environments. This is clearly possible, since they are allowed to migrate. Although they eat all their resources in one patch, there is enough food in the other patch to sustain them. Once the resource is exhausted in one patch it cannot recover, because prey cannot be imported from the other one (since the prey do not migrate). For $Y^{(2)}$ to be achievable (the prey disappear from the first patch), the stability condition (4.12) amounts to a suitable lower bound on the predator migration rate from the second patch into the first - i.e.

$$
\frac{k_{2} a e\left(\mu_{1}+n_{21}\right)^{2}}{k_{2} a e\left(\mu_{1}+n_{21}\right)-\sigma}<n_{12},
$$

where the denominator is positive in view of the feasibility condition (4.2). This makes sense, because if more predators enter the first patch they will increase the grazing rate on the prey, and ultimately wipe them out. A kind of dual result holds for the other patch for the prey-free equilibrium $Y^{(4)}$. Despite the fact that prey cannot migrate and thereby try to escape from voracious predators, it is notable that in this case the predators may indeed 
get extinguished, since equilibrium $Y^{(5)}$ is always feasible and only the stability conditions (4.13) need to be satisfied.

In system (5.1), when only the prey can migrate, the predators can be eliminated in just one patch while the prey thrive everywhere (equilibria $Z^{(2)}$ and $Z^{(3)}$ ). In this case, the predators are bound to disappear from at least one environment. Curiously enough, they may also be wiped out altogether as the prey only equilibrium $Z^{(1)}$ is always feasible, and only its stability conditions (5.5) need to be satisfied. The predators disappear from one patch only if the prey carrying capacity in the other patch is large enough, since from the feasibility condition (5.2) of (say) the former case $Z^{(2)}$ it follows

$$
k_{2}>\frac{r \mu_{2}}{a e\left[r+\frac{m_{21} k_{1} a e}{2 r \mu_{2}}\left(r-m_{21}+\sqrt{\left(r-m_{21}\right)^{2}+4 m_{12} \frac{r \mu_{2}}{k_{1} a e}}\right)-m_{12}\right]} .
$$

This result makes sense because, if the second environment is very rich in food for the prey, the prey tend to migrate there so the resources the predators need in the first patch are easily exhausted. Thus the predators starve there, since they are not allowed to migrate away from their environment. A similar result holds of course for $Z^{(3)}$. Thus the patch in which predators disappear acts as a refuge for the prey, where they can reproduce in safety and therefore guarantee (via migration) the survival of the ecosystem in the other patch too.

\section{Acknowledgments}

We thank the Max Planck Institut für Physik Komplexer Systeme in Dresden, Germany for facilities provided during a visit by one of us (EV) when this paper was completed.

\section{References}

[1] L. K. BAy, M. J. CALEY, R. H. CROZIER, Meta-population structure in a coral reeffish demonstrated by genetic data on patterns of migration, extinction and re-colonization, BMC Evolutionary Biology 8:248 (2008), doi:10.1186/1471-2148-8-248.

[2] H. L. Black, A North Temperate Bat Community: Structure and Prey Populations, Journal of Mammalogy 55 (1974), 138-157.

[3] V. Brack, JR., R. K. LaVAL, Food habits of the Indiana bat in Missouri, Journal of Mammalogy 66 (1985), 308-315.

[4] R. J. Cowie, S. A. Hinsley, Feeding ecology of great tits (Parus major) and blue tits (Parus caeruleus), breeding in suburban gardens, Journal of Animal Ecology 57 (1988), 611-626.

[5] J. T. Cronin, Movement and spatial population structure of a prairie planthopper, Ecology 84 (2003), 1179-1188.

[6] T. Dewey, L. Ballenger (1999) "Ovis canadensis" (On-line), Animal Diversity Web, http://animaldiversity.ummz.umich.edu/site/accounts/information/Ovis_canadensis.html.

[7] M. Drechsler, K. Johst, Rapid viability analysis for metapopulations in dynamic habitat networks, Proceedings of the Royal Society B: Biological Sciences 277 (2010), 1889-1897. 
[8] A. El Abdllaoui, P. Auger, B. Kooi, R. Bravo de la Parra, R. Mchich, Effects of densitydependent migrations on stability of a two-patch predator - prey model, Mathematical Biosciences 210 (2007), 335-354.

[9] E. J. Gustafson, R. H. Gardner, The effect of landscape heterogeneity on the probability of patch colonization, Ecology 77 (1996), 94-107.

[10] R. J. Gutiérrez, S. Harrison, Applying metapopulation theory to spotted owl management: A history and critique, in D. R. McCollough (Ed.) Metapopulations and Wildlife Conservation, Washington: Island Press, 167-185, 1996.

[11] I. HANSKI, Single-species spatial dynamics may contribute to long-term rarity and commonness, Ecology 66 (1985), 335-343.

[12] I. Hanski, A. Moilanen, T. Pakkala, M. Kuussaari, Metapopulation persistence of an endangered butterfly: A test of the quantitative incidence function model, Conservation Biology 10 (1996), 578-590.

[13] P. Kareiva, Population dynamics in spatially complex environments: Theory and data, Philosophical Transactions of the Royal Society of London B 330 (1990), 175-190.

[14] Z. Z. LI, M. GAO, C. Hui, X. Z. HAN, H. SHI, Impact of predator pursuit and prey evasion on synchrony and spatial patterns in metapopulation, Ecological Modelling 185 (2005), 245-254.

[15] H. Malchow, S. Petrovskit, E. Venturino, Spatiotemporal Patterns in Ecology and Epidemiology, CRC, Boca Raton, 2008.

[16] A. Mollanen, I. HANSKI, Habitat destruction and competitive coexistence in a spatially realistic metapopulation model, Journal of Animal Ecology 64 (1995), 141-144.

[17] A. Mollanen, A. Smith, I. Hanski, Long-term dynamics in a metapopulation of the American pika, American Naturalist 152 (1998), 530-542.

[18] K. L. RyAlL, L. FAHRIG, Response of predators to loss and fragmentation of prey habitat: A review of theory, Ecology 87 (2006), 1086-1093.

[19] G.Q. Sun, Z. Jin, Q.X. LIU, L. LI, Dynamical complexity of a spatial predator — prey model with migration, Ecological Modelling 219 (2008), 248-255.

[20] E. Venturino, Simple metaecoepidemic models, Bulletin of Mathematical Biology, 73 (2011), 917-950.

[21] A. A. Yakubu, M. J. Fogarty, Spatially discrete metapopulation models with directional dispersal, Mathematical Biosciences 204 (2006) 68-101.

[22] J. A. WIENS, Wildlife in patchy environments: metapopulations, mosaics, and management, in D. R. McCullough (Editor) Metapopulations and Wildlife Conservation, Washington: Island Press, 53-84, 1996.

[23] J. A. Wiens, Metapopulation dynamics and landscape ecology, in I. A. Hanski, M. E. Gilpin (Eds.) Metapopulation Biology: Ecology, Genetics, and Evolution, San Diego: Academic Press, 43-62, 1997.

[24] J. Wu, Modeling dynamics of patchy landscapes: linking metapopulation theory, landscape ecology and conservation biology, in Yearbook in Systems Ecology (English edition) Beijing: Chinese Academy of Sciences, 1994. 